\title{
Coal dust exposure triggers heterogeneity of transcriptional profiles in mouse pneumoconiosis and Vitamin D remedies
}

Min Mul, $2,3,4+$, Bing Lij ${ }^{3+}$, Yuanjie Zou ${ }^{3}$, Wenyang Wang ${ }^{3}$, Hangbing Cao ${ }^{3}$, Yajun Zhang ${ }^{1,2,3,4}$, Qixian Sun ${ }^{3}$, Haoming Chen ${ }^{3}$, Deyong Ge $\mathrm{G}^{1,2,3,4}$, Huihui Tao ${ }^{1,2,3,4}$, Dong Hu ${ }^{1,2,3,4}$, Liang Yuan 1,2,3,4* , Xinrong Tao ${ }^{1,2,3,4^{*}}$ and Jianhua Wang ${ }^{1,5^{*}}$

\begin{abstract}
Background: Coal dust particles (CDP), an inevitable by-product of coal mining for the environment, mainly causes coal workers' pneumoconiosis (CWP). Long-term exposure to coal dust leads to a complex alternation of biological processes during regeneration and repair in the healing lung. However, the cellular and complete molecular changes associated with pulmonary homeostasis caused by respiratory coal dust particles remain unclear.

Methods: This study mainly investigated the pulmonary toxicity of respirable-sized CDP in mice using unbiased single-cell RNA sequencing. CDP $(<5 \mu \mathrm{m})$ collected from the coal mine was analyzed by Scanning Electron Microscope (SEM) and Mass Spectrometer. In addition, western blotting, Elisa, QPCR was used to detect gene expression at mRNA or protein levels. Pathological analysis including HE staining, Masson staining, immunohistochemistry, and immunofluorescence staining were performed to characterize the structure and functional alternation in the pneumoconiosis mouse and verify the reliability of single-cell sequencing results.

Results: SEM image and Mass Spectrometer analysis showed that coal dust particles generated during coal mine production have been crushed and screened with a diameter of less than $5 \mu \mathrm{m}$ and contained less than 10\% silica. Alveolar structure and pulmonary microenvironment were destroyed, inflammatory and death (apoptosis, autophagy, and necrosis) pathways were activated, leading to pneumoconiosis in post 9 months coal dust stimulation. A distinct abnormally increased alveolar type 2 epithelial cell (AT2) were classified with a highly active state but reduced the antimicrobial-related protein expression of LYZ and Chia1 after CDP exposure. Beclin1, LC3B, LAMP2, TGF- $\beta$, and MLPH were up-regulated induced by CDP, promoting autophagy and pulmonary fibrosis. A new subset of macrophages with M2-type polarization double expressed MLPH +/CD206 + was found in mice having pneumoconiosis but markedly decreased after the Vitamin D treatment. Activated MLPH +/CD206 + M2 macrophages secreted TGF- $\beta 1$ and are sensitive to Vitamin D treatment.
\end{abstract}

*Correspondence: yuanl_1960@sina.com; xrtao1116@outlook.com; jianhuaw2007@qq.com

${ }^{\dagger} \mathrm{Min} \mathrm{Mu}$ and Bing Li have contributed equally to this work.

${ }^{4}$ Anhui Province Engineering Laboratory of Occupational Health and Safety, Anhui University of Science and Technology, Huainan, China

${ }^{5}$ Cancer Institute, Fudan University Shanghai Cancer Center, Fudan University, Shanghai, China

Full list of author information is available at the end of the article permits use, sharing, adaptation, distribution and reproduction in any medium or format, as long as you give appropriate credit to the original author(s) and the source, provide a link to the Creative Commons licence, and indicate if changes were made. The images or other third party material in this article are included in the article's Creative Commons licence, unless indicated otherwise in a credit line to the material. If material is not included in the article's Creative Commons licence and your intended use is not permitted by statutory regulation or exceeds the permitted use, you will need to obtain permission directly from the copyright holder. To view a copy of this licence, visit http://creativecommons.org/licenses/by/4.0/. The Creative Commons Public Domain Dedication waiver (http://creativeco mmons.org/publicdomain/zero/1.0/) applies to the data made available in this article, unless otherwise stated in a credit line to the data. 
Conclusions: This is the first study to reconstruct the pathologic progression and transcriptome pattern of coal pneumoconiosis in mice. Coal dust had obvious toxic effects on lung epithelial cells and macrophages and eventually induced pulmonary fibrosis. CDP-induced M2-type macrophages could be inhibited by VD, which may be related to the alleviation of the pulmonary fibrosis process.

Keywords: Single-cell RNA sequencing, Alveolar regeneration, Coal dust pulmonary disease, Epithelial cells, Macrophage subset phenotype activation, Pulmonary toxicity

\section{Introduction}

Coal remains the primary source of electricity $(60 \%)$ and iron production (40\%) globally [1]. Coal dust hazards in coal mining have been studied continuously by the WHO (World Health Organization) and governments of many countries. As of 2018, pneumoconiosis accounted for $90 \%$ of occupational diseases reported in China's occupational health status reports, with 200 million people still at risk of occupational health exposure [2]. The rates of pneumoconiosis in Australia have started rising [3]. Many studies have clearly shown that coal particles have cytotoxicity, pro-inflammatory, pro-fibrotic properties, and a complex progress mechanism [4-6]. The most extensive study on pneumoconiosis in the U.S. revealed that respirable dust from coal miners caused progressive massive fibrosis (PMF) even after dust exposure has ceased [7, 8]. Mine Safety and Health Administration (MSHA) issued new regulations [9] for respirable coal dust concentration limit (from 2.0 to $1.5 \mathrm{mg} / \mathrm{m}^{3}$ ). However, the prevalence rate continued to increase, with some regions such as the central Appalachian area seeing a sixfold increase [10]. There is no effective treatment for coal workers' pneumoconiosis.

Continued exposure to high concentrations of coal dust may lead to an inability to remove accumulated dust particles, resulting in structural damage to the alveoli [11]. Alveolar epithelial cells are the primary functional unit for $\mathrm{O}_{2}$ exchange and the first point of contact for inhaled particles [11]. Successful alveolar epithelial repair and functional recovery require the proliferation and differentiation of alveolar type 2 stem/progenitor cells (AT2). During the regeneration process, alveolar epithelial cells have been reported to have dysplasia or developmental delays in repair [12], which is associated with many chronic lung diseases [13]. Commonly, inflammatory monocytes and pulmonary macrophages are the keys to lung repair and fibrosis regulators. Lung macrophages in healthy adults are divided into mononuclear mesenchymal macrophages and tissue-resident macrophages that develop from embryonic precursors. Abnormal tissue niches can lead to the development of abnormally differentiated tissue-resident macrophages [14]. Alveolar macrophages (AMs), the central target cells of coal dust during phagocytosis and clearance, are related to oxidative stress and the activation of the innate immune system [15]. Exogenous pathways regulate apoptosis and the release of fibrotic factors from AMs. TNF- $\alpha$, IL 6, and IL $1 \beta$ are recognized pro-inflammatory factors released by macrophages [16]. VD3 can significantly inhibit activation of the TGF- $\beta$ signaling pathway and up-regulation of fibronectin and collagen expression [11, 12].

The diversity of macrophages differentiates in coal pneumoconiosis, and the long-term coal dust-induced alveolar inflammatory mechanisms are worth studying.

Single-cell RNA sequencing (scRNA-Seq) technology can identify cell type-specific markers and provide definitions and functions of cell types at the molecular level. For example, Kyler et al. created a complete molecular cell map of the adult lung using scRNA-Seq, which peaked in the genome-wide expression profile of the lung [17]. The entire life cycle of alveolar type 2 cells was revealed during the maturation of bipotential progenitor cells in two alveolar lineages [18]. Recently, it was found that mouse embryonic lung tissue has rich progenitor cells with different ecological niches during early development [19]. Interestingly, the activation of mTORC1 in the lung stroma resulted in female-like changes in stromal and epithelial cells and decreased lung function [20]. In addition, many aging-related lung markers, including increased cholesterol biosynthesis in type 2 lung cells, adipose fibroblasts, and changes in the relative frequency of airway epithelial cells, are discovered [21]. The molecular mechanism of interstitial lung disease, which is often associated with degenerative changes in lung function, has been elucidated by scRNA-Seq. However, the diversity of macrophages differentiates in coal pneumoconiosis, and the long-term coal dust-induced alveolar inflammatory mechanism remains unclear.

Macrophages show diversity and adaptability in cell differentiation, phenotype, and function in response to lung disease and maintaining homeostasis. The heterogeneity of macrophages in bleomycin-induced pulmonary fibrosis in mice suggests that $\mathrm{CX} 3 \mathrm{CR} 1^{+}$SiglecF ${ }^{+}$transition macrophages are localized to the fibrosis niche and have a fibrotic role in vivo [22]. Two independent interstitial macrophages (IM) subsets: Lyve $1{ }^{\mathrm{lo}} \mathrm{MHCII}{ }^{\text {hi }} \mathrm{CX} 3 \mathrm{CR} 11^{\text {hi }}$ (Lyve $1^{\mathrm{lo}} \mathrm{MHCII}{ }^{\mathrm{hi}}$ ) and Lyve $1^{\mathrm{hi}} \mathrm{MHCII}{ }^{\mathrm{lo}} \mathrm{CX} 3 \mathrm{CR} 1^{\mathrm{lo}}$ $\left(\right.$ Lyve ${ }^{\text {hi }}{ }^{M H C I I}{ }^{\mathrm{lo}}$ ) mononuclear derived IMs, have unique 
gene expression profiles, phenotypes, functions, and localization [23]. Furthermore, based on a new mouse model of macrophage depletion (Slco2b $1^{\text {flox/DTR }}$ ), the loss of Lyve $^{\text {hi }}{ }^{\text {MHCII }}{ }^{\text {lo }}$ IMs exacerbates experimental pulmonary fibrosis [23]. The crosstalk between alveolar macrophages and lung epithelial cells at the single-cell level can broaden our understanding of alveolar epithelial repair. Although bleomycin-induced IPF changes in cell types, including activated macrophages, fibroblasts, and proliferative epithelial cells, have been explored using scRNA-Seq. However, few studies related to coal dust pneumoconiosis have been reported [22, 24, 25], the Single-cell heterogeneity, the regulatory mechanism, and the Vitamin D treatment for coal dust pneumoconiosis have not been studied. Vitamin D deficiency occurs in $90 \%$ of cases of pulmonary fibrosis [26, 27], especially in patients with coal workers' pneumoconiosis who have lower Vitamin D levels than healthy controls [28, 29]. Poor Vitamin D status is associated with pulmonary fibrosis [30, 31], whereas Vitamin D protects against particles-caused lung injury through induction of autophagy in an Nrf2dependent manner, and it reduces lung injury and promotes tissue repair [32].

Here, an in-depth analysis of mouse lung macrophages and epithelial cells in 9-month post-coal exposure mice was performed, revealing the heterogeneity between the two populations in response to coal dust exposure using scRNA-Seq. In addition, the death signal analysis demonstrated the central roles of apoptosis-related pathways in death reprogramming in coal mice pneumoconiosis (CMP). Finally, cellular metabolism and communication landscapes were established within the single-cell resolution to explore better the interactions between AMs and epithelial cells in coal workers' pneumoconiosis (CWP). Our findings will enrich the understanding of the cellular and molecular differences between normal and CMP lung tissues.

\section{Material and methods \\ Chemicals and reagents}

We used Vitamin $\mathrm{D}_{3}\left[1,25(\mathrm{OH})_{2} \mathrm{D}_{3} ; \mathrm{VD}_{3}\right]$ (Sigma, product code: 740551, CAS: 128723-16-0, purity: 96\%), and Vitamin $\mathrm{D}_{3}$ (Macklin Biochemical, product code: C804669, CAS: $67-97-0$, purity: $98 \%$ ) for the in vitro and in vivo experiments, respectively.

\section{Coal dust preparation}

We used the Gillian dust sampler to collect respirable coal dust in a coal mine in the Anhui Province, China. The distribution of particle size was detected by the Malvern laser particle size analyzer. Different particle size fractions were found $(1 \mu \mathrm{m}: 8.42 \%, 1-5 \mu \mathrm{m}: 68.42 \%$, and $<5 \mu \mathrm{m}$ ) and verified by SEM (Zeiss, EVO) (Fig. 1A, B).
Particle size less than 5 microns accounted for $76.84 \%$ of the total particle number of dust samples. Each particle contained less than $10 \%$ silica revealed by ICP-MS (Agilent 7700) (Fig. 1C). Coal dust was weighed and autoclaved at $121{ }^{\circ} \mathrm{C}$ for $30 \mathrm{~min}$ before drying. Endotoxin detection was performed using the LAL test. The results showed that endotoxin was negative, $<0.01 \mathrm{EU} / \mathrm{ML}$. Then, the coal dust was suspended in phosphate buffer saline (PBS) and sonicated for $15 \mathrm{~min}$ to ensure a uniform suspension before use.

\section{Animal husbandry}

Male C57BL/6 mice [license number SCXY (Su) 20110003] were bought from the Changzhou Cavion Experimental Animal Co. The mice were raised in cyclic light with free access to food and water. All animal experiments in the study followed the ARRIVE guidelines. All procedures were conducted following the guidelines described in the National Institutes of Health's Guide for the Care and Use of Laboratory Animals (NIH Publication No. 8023, revised 1978) and were accepted by the Institutional Animal Care and Use Committee of the Anhui University of Science and Technology.

Mice $(\mathrm{N}=42)$ aged 6-8 weeks were assigned randomly to three groups: control group (vehicle), coal group, and Vitamin $\mathrm{D}_{3}$ group, each consisting of 14 mice. After two weeks of adaptation mice weight were $22.55 \pm 1.087 \mathrm{~g}$. A time-escalating study of 3,6 , and 9 months was performed to evaluate the in vivo toxicity of coal dust. Coal dust $(20 \mathrm{mg} / \mathrm{ml} / 60 \mu \mathrm{l}$ vs. saline) was administered intranasally into mouse lungs twice per week for four weeks with slight anesthetization [33]. Vitamin D3 was diluted by anhydrous ethanol and PBS and injected intraperitoneally in a volume of $0.1 \mathrm{~mL}$ three times per week at a dose of $400 \mathrm{IU} / \mathrm{Kg}$ until the experiment was completed.

\section{Hematoxylin and eosin (HE), Masson, and immunohistochemistry (IHC) staining}

Mouse lung tissues for histological analysis were prepared through paraffin embedding or cryopreservation followed by formalin fixation. HE (Solarbio, Beijing, China) and Masson (Solarbio, Beijing, China) staining was performed on $5 \mu \mathrm{m}$-thick sections, and $10 \mu \mathrm{m}$-thick free-floating frozen sections were stained for double immunofluorescence staining. Roderick J's method [34] was used to conduct pathology score of lung injury and Ashcroft's method [35] was used to conduct fibrosis score. Deparaffinized sections were pretreated with heat-based antigen retrieval reagents and blocking reagents to prevent the non-specific binding of antibodies to tissue. Rabbit monoclonal anti-EMR1 (Proteintech, Wuhan, China), anti-MLPH (Proteintech, Wuhan, China), anti-LC3B (Abcam, Shanghai, 


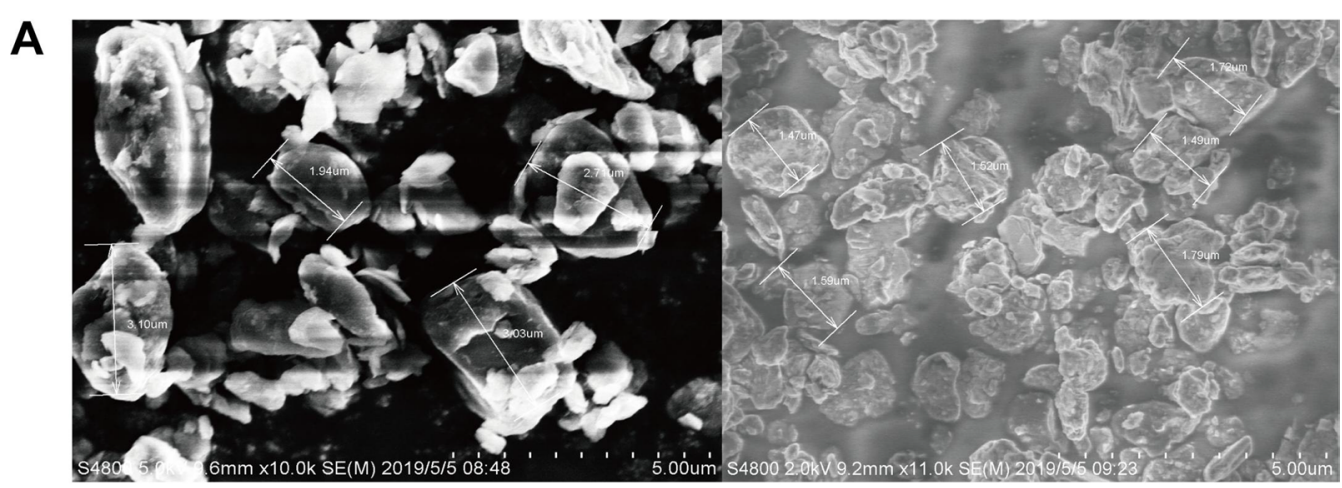

B
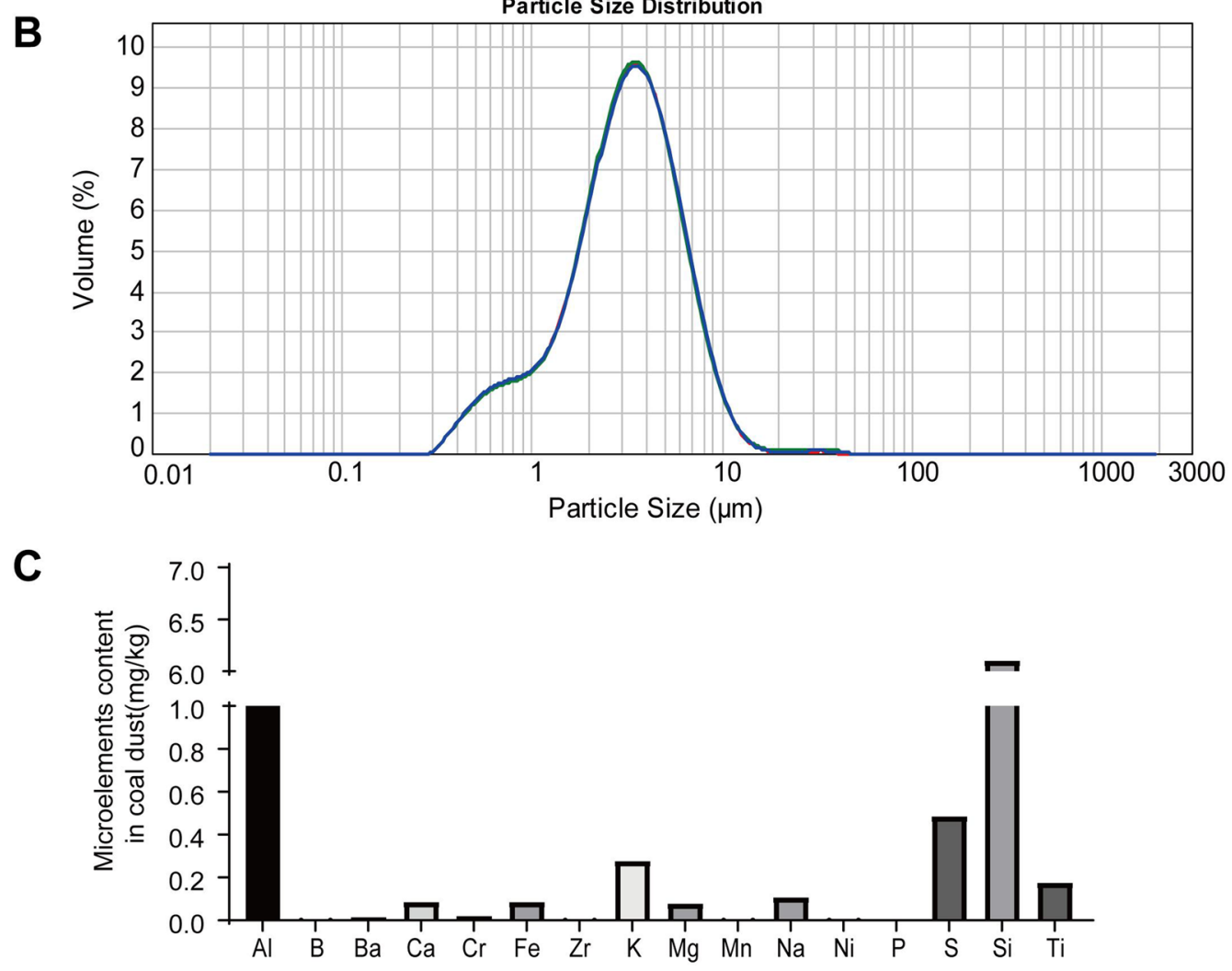

Fig. 1 The chemical composition and particle size of coal dust are involved in this experiment. A The image of coal dust particles under scanning electron microscopy. All the particles are symmetrical, and the particle size distribution is narrow and has low silica content. B The distribution of coal dust particles is shown in the graph. C The chemical composition of coal dust particles was analyzed and presented in the table

China), and anti-CD206 (Proteintech, Wuhan, China) were used as the primary antibody. Horse anti-rabbit IgG Polymer Detection Kit, horse anti-mouse IgG Polymer Detection Kit (Vector, San Francisco, CA, USA) were applied before chromogenic substrate (Vector, San Francisco, CA, USA) was added. The nucleus was counterstained with hematoxylin. Alexa Fluor ${ }^{\circledR} 488$ conjugate anti-mouse IgG (Cell Signaling Technology, Danvers, MA, USA), and Alexa Fluor ${ }^{\circledR} 594$ Anti-rabbit IgG (Abcam, Shanghai, China) were chosen for indirect double staining. DAPI (Beyotime, Shanghai, China) were counterstained for the nucleus. The sections were mounted with an antifade medium and observed under an Olympus FV3000 fluorescence microscope (Olympus, Tokyo, Japan), with all parameters (pinhole, contrast, and brightness) constant.

\section{Cell culture}

Vitamin D3 can't be converted into the active form $1,25(\mathrm{OH}) 2 \mathrm{D} 3$ by cultured cells. RAW264.7 cells 
were incubated with either $200 \mu \mathrm{g} / \mathrm{mL}$ coal dust, $1.2 \times 10^{-10} \mathrm{mg} / \mathrm{mL} 1,25(\mathrm{OH})_{2} \mathrm{VD}_{3}$, or a mixture of $200 \mu \mathrm{g} /$ $\mathrm{mL}$ coal dust and $1.2 \times 10^{-10} \mathrm{mg} / \mathrm{mL} 1,25(\mathrm{OH})_{2} \mathrm{VD}_{3}$, respectively, for $24 \mathrm{~h}$. Subsequently, the cells were fixed with a $4 \%$ paraformaldehyde solution for $15 \mathrm{~min}$. AntiMLPH as the primary antibody, Alexa Fluor ${ }^{\circledR} 594$ conjugated goat anti-rabbit secondary antibody (Invitrogen, CA, USA) were used. DAPI dye stained for $10 \mathrm{~min}$. Images were taken using a confocal laser scanning microscope (Olympus, Tokyo, Japan).

\section{Quantitative real-time PCR (qPCR) assay}

Raw 264.7 cells were harvested, and total RNA was isolated using Trizol extraction (Thermo Fisher Scientific, Waltham, MA, USA). For cDNA synthesis, a $5 \times$ All-InOne RT MasterMix kit (abm, Vancouver, Canada) was used. The real-time polymerase chain reaction was performed on a QuantStudio three Real-Time PCR Systems (Thermo Fisher Scientific, Waltham, MA, USA). For one amplification reaction, the reaction volume was kept at $10 \mu \mathrm{l}$ by including $1 \mu \mathrm{l}(10 \mathrm{ng})$ of cDNA, $5 \mu \mathrm{l}$ of GoTaq ${ }^{\circledR}$ qPCR Master Mix (Promega, Madison, WI, USA), $0.4 \mu \mathrm{l}$ of primer $(10 \mu \mathrm{M})$, and DNase-free water. The primers are as follows: TGF- $\beta$, (FW)5-TGACGTCACTGGAGT TGTACGG-3, (RV)5'-GGTTCATGTCATGGATGG TGC-3'; GAPDH, (FW)5'-CCTCGTCCCGTAGAC AAAATG-3', (RV) $5^{\prime}$-TGAGGTCAATGAAGGGGT CGT-3'. The relative abundance of transcripts of each gene was calculated according to the comparative $\Delta \Delta C T$ method.

\section{ELISA}

Raw 264.7 cells were cultured with coal dust for $24 \mathrm{~h}$, the supernatants were collected, and the concentrations of TGF- $\beta 1$ were measured using ELISA kits (Catalog: RK00057; ABclonal, Wuhan, China) according to the manufacturer's protocol.

\section{Western blot assay}

Raw 264.7 cells treated with coal dust $(200 \mu \mathrm{g} / \mathrm{mL})$ and $1.25(\mathrm{OH})_{2} \mathrm{VD}_{3}\left(1.2 \times 10^{-10} \mathrm{mg} / \mathrm{mL}\right)$ were harvested, and cell lysis was obtained in RIPA buffer (Beyotime, Shanghai, China). Supernatants were collected, followed by centrifuging at a speed of 10,000 rpm for $10 \mathrm{~min}$, and the protein concentration was determined using a BCA protein concentration determination kit (Beyotime, Shanghai, China). Next, $30 \mu \mathrm{g}$ of the protein sample was loaded on an SDS-PAGE gel and electrophoresed. The electrophoresed proteins were transferred onto a polyvinylidene difluoride (PVDF) membrane (Thermo Fisher Scientific, Waltham, MA, USA). After blocking, MLPH antibody or GAPDH (Cell Signaling Technology, Danvers, MA, USA) was added. Chemiluminescence was then used to detect the proteins with an ECL developer (Thermo Fisher Scientific, Waltham, MA, USA). Finally, the protein expression levels were quantitated using ImageJ software.

\section{Quantification and statistical analysis}

Statistical analyses were performed using the SPSS Statistics for Windows, version 11.0 (SPSS Inc., Chicago, IL, USA), and statistical significance was set at $p<0.05$. The data are presented as means \pm SEM. Statistical comparisons were performed using unpaired Student's $t$-tests for two-tailed $p$ values $\left(" p<0.05,{ }^{* * *} p<0.01\right.$, and $\left.{ }^{* * * *} p<0.001\right)$.

\section{Tissue processing}

Samples from the coal spots and adjacent normal $C 57 B L / 6$ mouse lung tissue from the same resection specimen were isolated and transported rapidly to the research facility. Each sample was subsequently minced on ice to less than 1-mm cubic pieces, followed by enzymatic digestion using DNase I (Worthington; $30 \mathrm{U} \mathrm{mL} \mathrm{1),}$ collagenase IV (Worthington; $195 \mathrm{U} \mathrm{mL}$ 1), collagenase I (Worthington; $10 \mathrm{U} \mathrm{mL} \mathrm{1),} \mathrm{and} \mathrm{30 \%} \mathrm{FBS} \mathrm{for} 1 \mathrm{~h}$ at $37^{\circ} \mathrm{C}$, with manual shaking every $5 \mathrm{~min}$. Then washing, cell resuscitation, lysis of red blood cells, filtration, cell count were as followed [36].

\section{Advanced analytical methods for biological information}

After Single-cell RNA sequencing [36] and Single-cell RNA-seq data preprocessing [37], we performed the following advanced bioinformatics analysis methods: subclusters analysis, differential gene expression analysis, Gene Ontology (GO), KEGG pathway analyses [38], Gene set variation analysis (GSVA) [39], Gene set enrichment analysis (GSEA) [40], Cell cycle analysis [41], Pseudotime analysis [42], Cell-cell communication analysis [43-45]. Additional details are provided in the Supplemental data.

\section{Results}

Successful establishment of coal mice pneumoconiosis by exposure to coal dust particles

By exposing mice to coal dust twice-weekly for four weeks, we established a pathological phenotype of coal pneumoconiosis in these mice. Using this model, we studied the severity of coal dust lung damage at three, six, and nine months post-exposure, mainly reflected in the injury and repair of alveolar structures (Fig. 2A). In the third month, coal dust was embedded in the spaces between lung tissues, and cell proliferation resulted in alveolar stroma thickening. The coal dust particle has been phagocytosed by macrophages and aggregated into a ball, with lymphocytes proliferating into nodules in the sixth month. Finally, in the ninth month, the coal dust 
particles were again dispersed in the alveolar area, and the alveolar structure was destroyed and discontinuous. Roderick J. pathological grading of lung injury showed that the scores of coal dust exposed mice increased $\left({ }^{* * * *} P<0.001\right)$ significantly at three, six and nine months (Additional file 5: Fig. S5A). The significant increase in AT2 cells and the activation of repair functions resulted in the extracellular matrix deposition. Epithelial wounds repaired by infiltrating immune cells are shown in Fig. 2B, as illustrated by the diagram.

These results suggested that this technique was a reliable method for modeling CMP, as it produced an asymmetrical distribution of coal dust on both sides of the lungs. In addition, the inflammatory injuries in the lungs presented a trend of severe, moderate, and mild from the hilum to the lung margin (Fig. 2C1-C4). These three levels presented obvious boundaries (as delineated by the red, yellow, and green lines), and the proportions of affected areas were $17 \%, 47 \%$, and $36 \%$, respectively (Fig. 2D). The progression of pulmonary fibrosis in these mice at three, six, and nine months was demonstrated by Masson staining (Fig. 2E). Experimental coal pneumoconiosis in the mice had been latent for an extended period of six months before the formation of significant fibrosis. However, nine months after coal dust exposure, there was significant interstitial pulmonary fibrosis with a darker toluidine blue margin, suggesting an idiopathic centripetal fibrosis-like progression. Pulmonary fibrosis score performed by Ashcroft criteria for grading lung fibrosis showed that the scores of coal dust exposed mice increased $\left({ }^{* * * *} P<0.001\right)$ significantly at three, six and nine months (Additional file 5: Fig. S5B). The collagenous volume fraction (CVF), which reflects fibrosis progression, was quantitatively assessed, as was the alveolar volume fraction, an indicator for lung function in mice. There was a significant increase in CVF in nine months after coal exposure $(p<0.001)$ (Fig. 2F). With the increase of CVF in the development of fibrosis, the alveolar volume fraction (AVF) of the mice decreased (Fig. 2G), indicating a decline in respiratory function. Moreover, the Penh value was higher and the values of Mv, F, Av, EF50, PIF, PEF, Rpef were lower $\left({ }^{*} P<0.05,{ }^{* *} P<0.01,{ }^{* * *} P<0.001\right)$ in the coal dust group compared with the control group (Additional file 5: Fig. S5D). This result indicated that the mice had increased respiratory resistance and decreased lung capacity at nine months after coal dust exposure. The mice weight decreased in the first month after coal dust exposure, but increased continually after coal dust ceased. However, the average weight of the coal dust group mice was lower than that of the control ones from eight to nine months (Additional file 5: Fig. S5C). At nine months, mice exposed to coal dust were subject to spontaneous death, a disease outcome associated with fibrosis progression.

\section{Classification of lung cells based on cell-type specific marker genes}

According to the experimental flow chart (Fig. 3A), singlecell suspensions of 9-month-exposed murine pulmonary cells were prepared. We isolated and sequenced 50,156 cells from whole lung cell suspensions of four male mice, including two vehicle control mice and two coal-exposed mice. We first cataloged mouse lung cell types in an unbiased manner using droplet-based scRNA-Seq on the $10 \times$ Genomics platform and performed analyses using a single-tube protocol with unique transcript counting through barcoding with UMIs. Then, the effects of UMI and mitochondrial gene content were examined (Fig. 3B). The clustering of cells was not affected by UMI or the mitochondrial gene content. Besides, genes positively correlated with mitochondria-encoded proteins were found associated with solute transport rather than cellular stress responses. After quality filtering, 50 million unique transcripts were obtained from 42,252 cells, in which more than 1,303 genes could be detected. Of these, 24,094 cells $(48 \%)$ originated from coal pneumoconiosis-infected lungs and 26,062 from control lungs (Additional file 8: Table S1). We performed dimensionality

\footnotetext{
(See figure on next page.)

Fig. 2 Dispersion of the inhaled coal dust into murine lungs and induction of lung fibrosis progression. The coal dust suspension was instilled via the nose, b.i.W., to accumulate $20 \mathrm{mg}$ coal dust weight. Mice were examined at 3th, 6th and 9th months post-exposure. A Representative histological images (H\&E) show the progress of pneumoconiosis tissue injury at 3,6, and 9 months post-exposure. Green arrowheads pointed to the AT2. B Schematic diagram depicting lung injury and repair during coal pneumoconiosis at 9 months post-exposure (AT1: alveolar epithelial cell type 1, AT2: alveolar epithelial cell type 2, AAT2: Activated alveolar epithelial cell type 2, CDPs: coal dust particles, FN: fibronectin, RFs: repair related factor, BEC: blood endothelial cells, ExCM: extracellular matrix). C1-C4 A representative sample of lung tissue showing a central-to-peripheral decline of lung interstitial cell density at 6 months, graded as severe (S), moderate (M), or light (L). D The lung section is divided into three regions, based on the density gradient of interstitial cells, representing severe, moderate, and mild damage. The proportion of the area of different lung interstitial cell density grades at the 6th month. E Representative histological images (Masson trichrome) showing pulmonary fibrosis progression in mice lungs at 3,6 , and 9 months after coal dust exposure (black arrows pointed to peripheral fibroblasts), $40 \times$ and $100 \times$ magnifications. F Collagenous volume fraction (CVF) at 3, 6, and 9 months, the area of collagen staining in lung tissue. G Alveolar volume fraction (AVF) at 3, 6, and 9 months, the area of the alveolar air cavity gradually shrinks. Both AVF and CVF were measured by free software Image J (NIH, http://rsbweb.nih.gov/ij/). The 40x, 200x, $400 \times$ visual field were used and Semi-quantification under 200x $\left({ }^{*} p<0.05,{ }^{* *} p<0.01,{ }^{* * *} p<0.001\right)$
} 


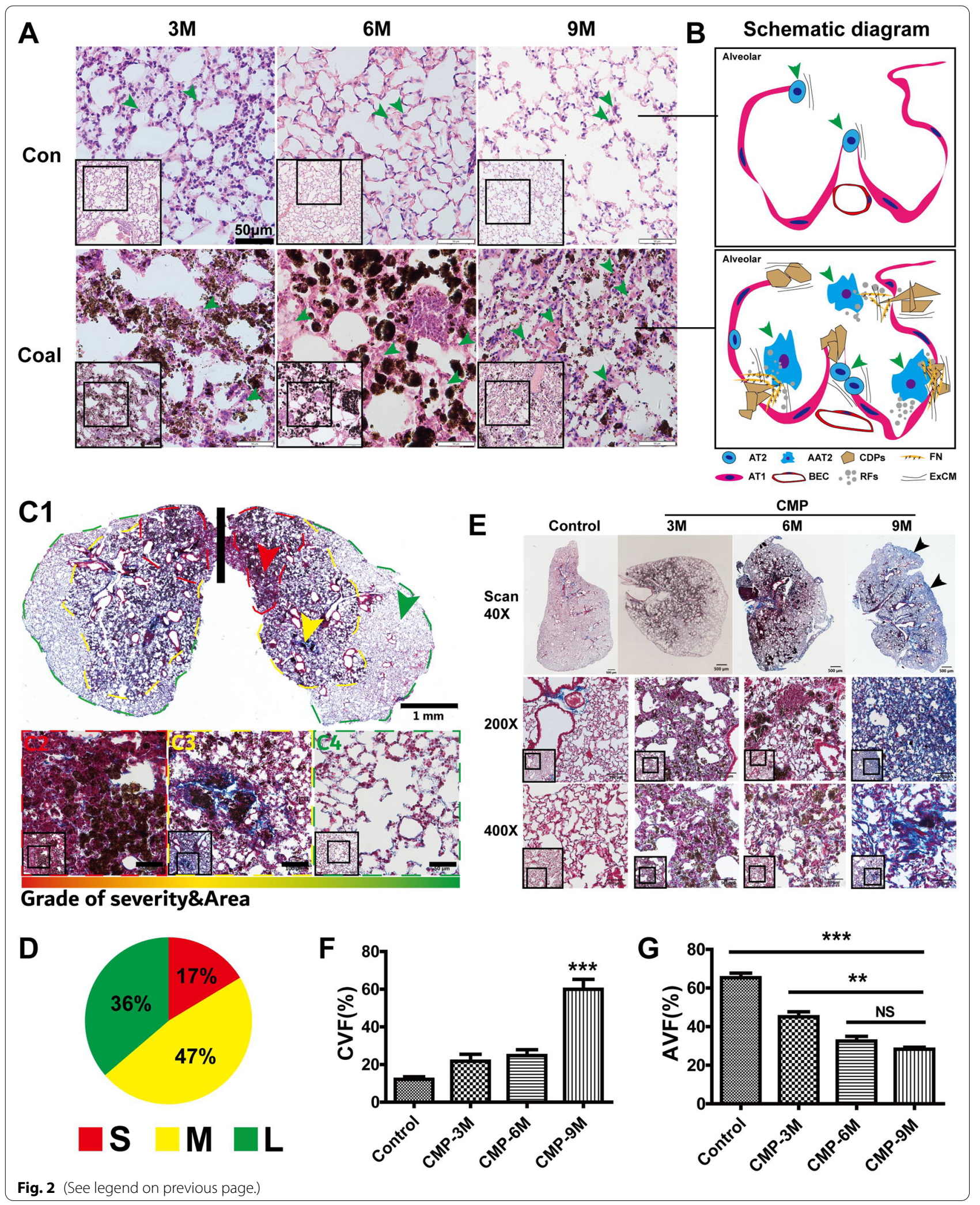




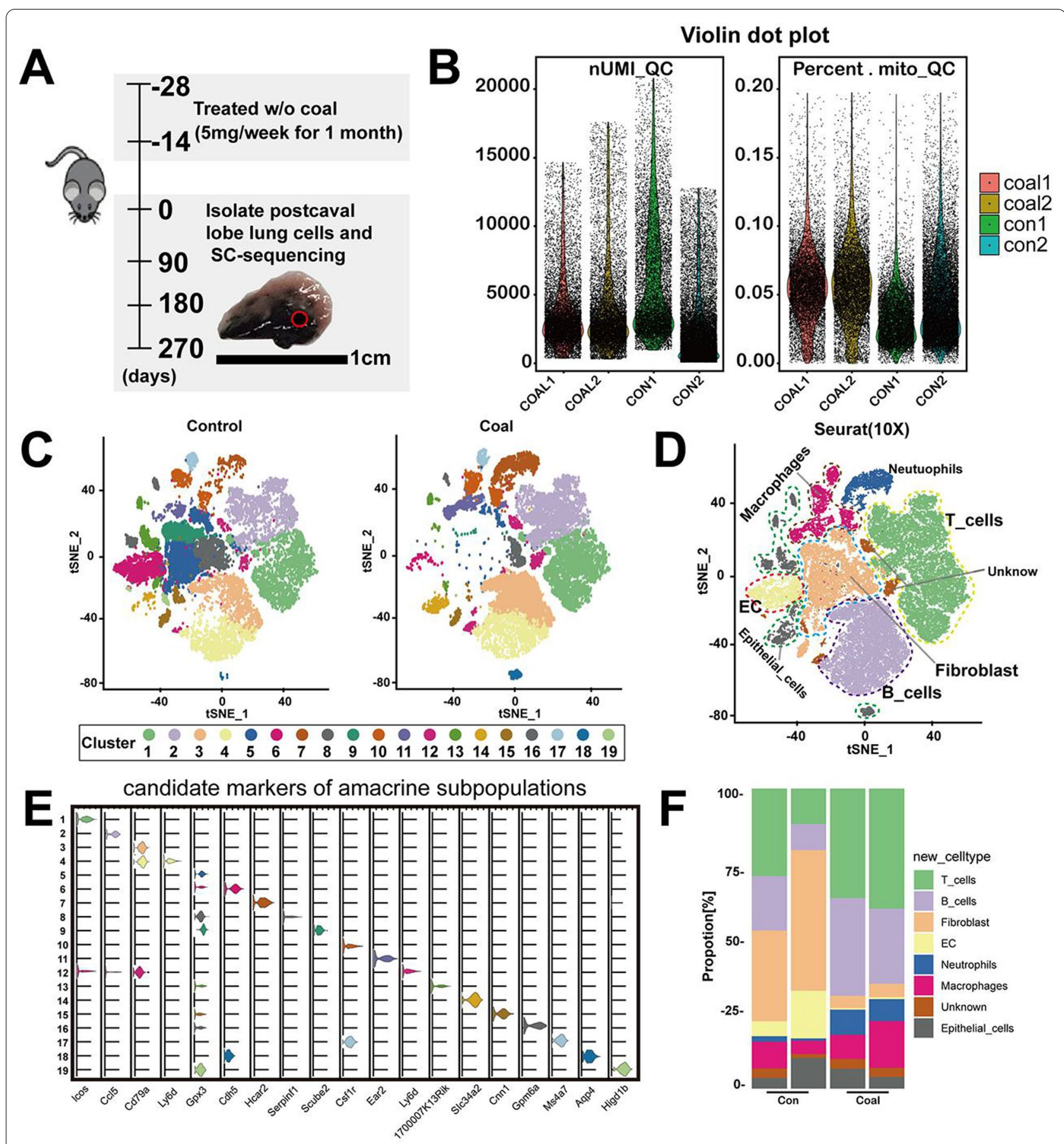

Fig. 3 Single-cell landscape in murine lungs exposed to coal dust. A Schematic of the experimental workflow used to define the pulmonary cells of 9-month-exposed mice. B Quality control of unique cell molecular identifiers (UMI) and mitochondria. C, D tSNE plot and clustering of 42,252 cells from coal-exposed mice $(n=2)$ and vehicle control mice $(n=2)$, classified into 19 clusters and seven distinct cellular types. E Violin plot showed the feature of vital lineage-associated in the mouse lung clusters. F Average proportion of eight main types of lung cells in coal dust-exposed lungs and control lungs

reduction with $\mathrm{t}$-SNE subspace alignment, followed by an unsupervised clustering assay (Fig. 3C). To characterize the heterogeneity of coal exposed-pulmonary cells at a higher resolution, we focused on seven clusters from 12,855 cells of coal-exposed and control lungs (Fig. 3D). 19 distinct cell clusters consisting of as few as 1186 cells 
to 15,665 cells per cluster were classified. The representative markers identified 19 clusters, including epithelial cells (Epcam, Krt18), endothelial cells (Pecam1, Cdh5), fibroblast cells (Dcn, Col1a1), macrophages (Cd114, Cd68, Csf1r), monocytes (CD14, CD300E), neutrophils (Arg2, Sorl1), and lymphocytes (CD79A, CD19, TRBC2) (Fig. 3C, E). All the clusters included cells from both coal dust-treated and untreated lungs, except for cluster 19, which included cells mostly from coal dust-treated lungs (Additional file 1: Fig. S1A). Two superclusters were categorized and focused: one consisted of clusters 13, 14, 16, and 18 with higher Krt18 and EPCAM expression, and the other consisted of clusters 10,11 , and 17 with higher CD14 and CD68 expression (Additional file 1: Fig. S1B\&C). Cluster 10 with the expression of CD14 and CD300E was recognized as proliferating cells. Clusters 11 and 17 expressed macrophage markers, such as CD14 and CD68. Cluster 18 expressed epithelial cell markers, such as ITGA1 and SCNN1G, and the highest level of Aqp4 (Fig. 3E). The cell-type composition distribution of each mouse was observed to differ substantially (Fig. 3F). As expected, immune cells, such as lymphocytes and macrophages, were the dominant cell type for coal pneumoconiosis.

\section{Intrinsic epithelial cell subpopulations underlying coal pneumoconiosis injury}

We detected 2,863 epithelial cells, including both epithelial and stromal cells. All the cells were re-analyzed, yielding 12 clusters. As expected, given the hypervascular nature of the lungs, the epithelial cells were less abundant (963 cells) in the coal pneumoconiosis group (Fig. 4A). Next, we attempted to identify marker genes for each cluster and assign them to known epithelial cell types. In many cases, the unbiased cluster identifier was a known cell-type-specific marker, such as surfactant protein C (SPC) for AT2 cells, Hopx for alveolar type I cells (AT1), and keratin 5 (KRT5) for basal cells
(Fig. 4E). Notably, we identified many additional markers, followed by some of the known markers. One set of 48 Clara cells was found in coal pneumoconiosis samples (cluster 10; marker genes Scgbla1 and MUC5b), as well as three sets of lymphatic epithelial cells $\left(\mathrm{PDPN}^{+}\right)$; two mainly were coal pneumoconiosis-derived (clusters 4 and 5; $\mathrm{AQP3}^{+}$and $\mathrm{AQP} 1^{+}$) and one controlled lung-derived (cluster 3; SCNN1G). To identify differentially expressed genes (DEGs) that may play an essential role in the defense response to coal dust in murine lungs, the CWP group (Additional file 8: Table S2) that inhaled coal dust was compared to the vehicle control group. 793 DEGs were identified using the standard FDR of $<0.01$ and foldchange of 1.5 , of which 330 were upregulated and 463 were downregulated (Fig. 4C). The downregulated genes included those involved in the heat shock protein family (Hspa1b, Hsp90aa1, and Hspa1a), calcium-regulated actin-modulating protein (Gsn, Mgp, and LIMCH1), and intercellular adhesion (Thbs1 and Vcam). The distribution of DEGs is illustrated using a volcano plot (Fig. 3C), where the red color represents elevated expression and blue represents decreasing expression levels. To visualize the gene expression profiles of the top 20 genes, we generated a heatmap using a ring diagram (Fig. 4B). To further tell the function of DEGs, 793 DEGs from mice with or without coal dust exposure were subjected to GO annotation (Fig. 4D). The results demonstrated that 5269 GO terms were annotated, including 3711 biological processes (9543 unigenes), 618 cell components (5,164 unigenes), and 940 molecular functions (3993 unigenes). Most of the differentially expressed genes were mainly involved in the biological processes of cells. The DEGs in this study were mainly involved in cellular processes (GO: 0098869), molecular function (GO: GO: 0032981), ribosomal function (GO: 1990932 and GO: GO: 0006412), and ATP biosynthetic processes (GO: 0006754), all of which indicate that numerous physiological and biochemical alternation in the epithelial

\footnotetext{
(See figure on next page.)

Fig. 4 Longitudinal single-cell RNA-seq reveals the heterogeneity of epithelial subtypes and alveolar epithelial regeneration after long-term exposure to coal dust. A The tSNE plot shows the changes in the distribution of the subtypes of epithelial cells 9 months after coal dust-mediated lung injury. B Differentially expressed genes (DEGs) from scRNA-seq were identified, and the top 50 DEGs are shown. C Volcano plots highlight differentially expressed genes in the coal dust-exposed and control pulmonary cells. Red dots indicate gene expression upregulation in coal dust-exposed mice, while blue dots indicate downregulation after coal exposure. Labels were added to DWFb1, Krt5, and the top 20 most DEGs. D Gene ontology (GO) classification provides putative gene functions for pulmonary cells in coal-exposed mice. E Single-cell annotation and unsupervised migration clustering identified nine subtypes of epithelial cells. $\mathbf{F}$ Based on the lung epithelial cell analysis, the annular heat map shows the significant DEGs out of the top 50 DEGs. The single-cell sequencing data revealed that these top 50 genes were differentially expressed in alveolar dual potent progenitor cells and type 2 alveolar epithelial cells. G CHIL1 and SFTPC overexpression in-coal exposed lungs were identified by immunohistochemical staining that compared the coal-exposed lung tissues to the control lungs. $\mathbf{H}$ The proliferating epithelial subsets expressed APOC1, CXCL15, and IL33, covering the primary epithelial cells with CHILA1, LYZ, and HBEGF. Cell markers were used to identify clusters as represented in the tSNE plot. I Using pseudotime ordering analysis, we successfully constructed the pulmonary epithelial cell lineage as a differentiation trajectory. Each branch shows a single-cell state. The top left plot is marked with developmental time, and the right plot is marked with cell states
} 


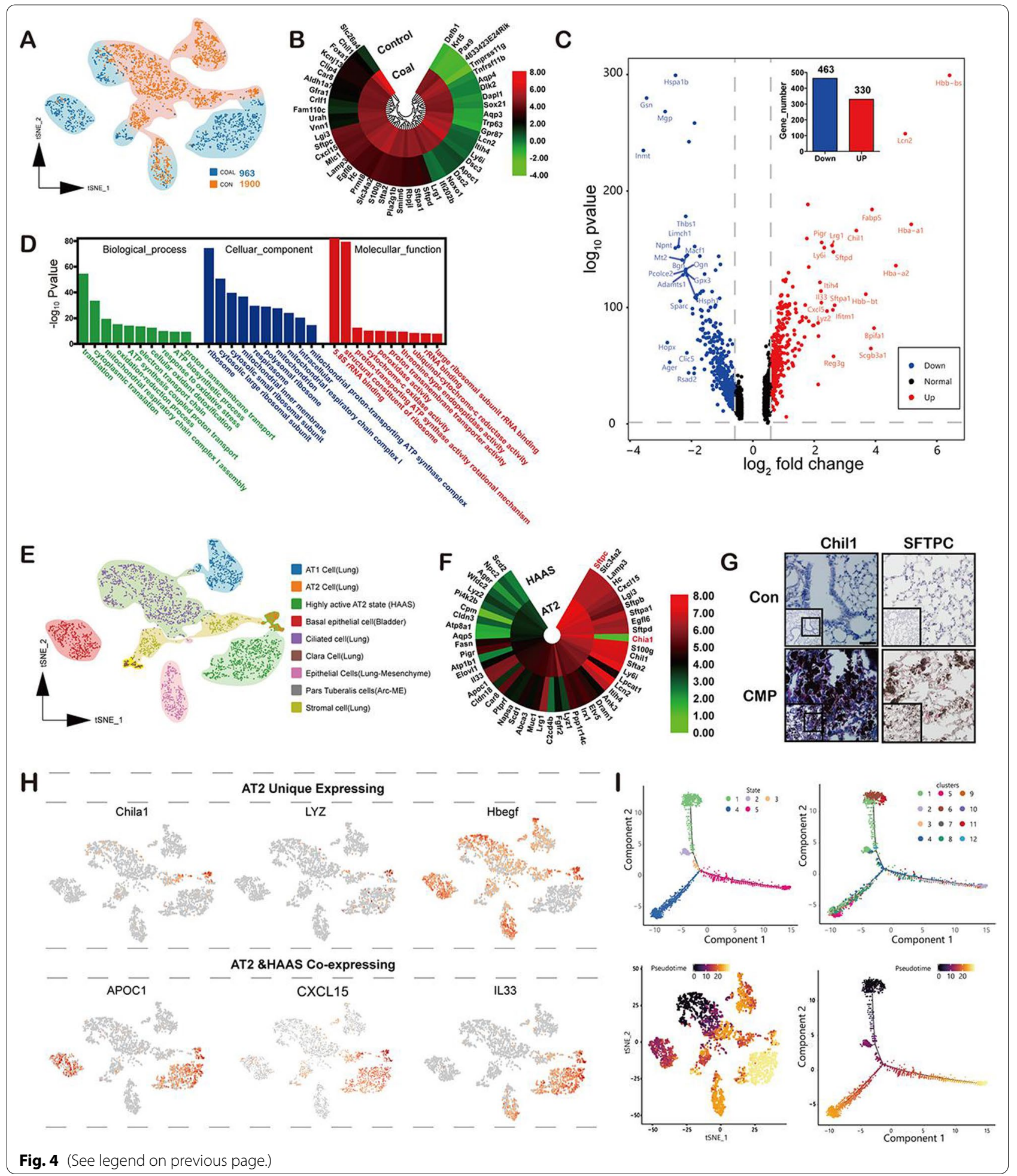

cells during coal dust inhalation into the lung, an important role in the coal dust defense response. According to the epithelial subtype identification results, we classified the epithelial cells into nine types. Compared with the control group, coal pneumoconiosis type II epithelial cells were more and had a significant difference in dimensionality reduction clustering, which was defined as a highly active AT2 state (HAAS) (Fig. 4E), and focused 
on the reprogramming of AT2 epithelial cells (cluster 2 and cluster 9). Next, a direct comparison of HAAS and normal AT2 epithelial cells was performed. We found that the antimicrobial-related protein expression of LYZ and Chia1 was missing in the AT2 epithelial cells of mice with coal pneumoconiosis (Fig. 4F). Remarkably, the total read counts were 7 - to 8- fold higher in HAAS than in normal AT2 epithelial cell clusters, accounting for $48 \%$ of the total coal pneumoconiosis epithelial cells (Additional file 8: Table S3). Using immunohistochemical analysis of independent coal pneumoconiosis samples for CHIL1, SFTPC, and HBEGF markers of clusters 2 and 9, we confirmed the presence of these cells as separate cellular entities, which were enriched in coal pneumoconiosis tissues (Fig. 4G). The analysis of hallmark gene expression signatures highlighted that, while the two-coal pneumoconiosis epithelial cell clusters showed some differences, most changes were between the control lung and coal pneumoconiosis-derived AT2 epithelial cells (Fig. 4H). In short, these results indicate that coal pneumoconiosis epithelial cells are remodeled to increase the number of new AT2 epithelial cells, thus contributing to coal pneumoconiosis regeneration and the repair of coal dustinjured epithelial tissue. Finally, to map cell types, states, and transitions during the regeneration of dynamic biological processes in coal pneumoconiosis, we performed a pseudotime analysis of the epithelial cells using Monocle. The data suggested two diverging cell fates, starting at clusters 6,8 , and 9, progressing toward clusters 1,5 , and 7 at one end, and cluster 2 at the other, with cluster 4 being a transitioning state spreading along the axis (Fig. 4I).

\section{Coal dust induces murine AT 2 cell apoptosis and reprogramming}

To verify the toxicity of coal dust in vitro, we used the mouse AT2 epithelial cell line MLE-12. MLE-12 cells grown on polylysine-coated slides in 24-well-plates were supplemented with a complete culture medium containing $10 \%$ FBS before they were exposed to $0 \mu \mathrm{g} / \mathrm{mL}$, $200 \mu \mathrm{g} / \mathrm{mL}, 400 \mu \mathrm{g} / \mathrm{mL}, 800 \mu \mathrm{g} / \mathrm{mL}$ and $1000 \mathrm{ug} / \mathrm{ml}$ coal dust for $24 \mathrm{~h}$. With the increase of exposure concentration, the inhibition of MLE-12 gradually increased and finally reached causing $50 \%$ inhibition of cell survival (CC50) at $1000 \mu \mathrm{g} / \mathrm{ml}$ (Additional file 6: Fig. S6A). Then we studied the effect of coal dust exposure time on cytotoxicity. At the highest concentration of which the cells were not significantly inhibited, i.e. $200 \mathrm{ug} / \mathrm{ml}$, the cell inhibition rate did not change within $36 \mathrm{~h}$ (Additional file 6: Fig. S6B). Meanwhile, we analyzed cell apoptosis using an annexin V/PI apoptosis detection kit under confocal laser microscopy. FITC-conjugated annexin V and propidium iodide (PI) staining were used to discriminate between early apoptotic and late apoptotic/necrotic cells (Fig. 5A). The MLE-12 cells treated with $200 \mu \mathrm{g} / \mathrm{mL}$ had the highest number of green-colored apoptotic cells after $24 \mathrm{~h}$ post-exposure relative to the $400 \mu \mathrm{g} / \mathrm{mL}$ or $800 \mu \mathrm{g} /$ $\mathrm{mL}$ groups, indicating that AT2 epithelial cells were sensitive to the stimulation of coal dust at a low dose. Conversely, cells treated with $400 \mu \mathrm{g} / \mathrm{mL}$ had the highest number of red-colored late apoptotic/necrotic cells, indicating that the limit of the lethal concentration of coal dust in AT2 cells was $400 \mu \mathrm{g} / \mathrm{mL}$ (Fig. 5B). Additionally, we verified AT2 apoptosis in vitro using the immunofluorescent staining of anti-caspase 3 antibody (green fluorescence) and found that CCP3 positive AT2 cells in the coal dust group had obvious nuclear pyknosis and fragmentation (arrowhead) (Fig. 5C).

We believe that epithelial cells are the first line of defense (as opposed to immune cells), and any damage to them causes pro-inflammatory factor release, which initiates the body's immune response. The apoptosis/necrosis pathways were further analyzed following the in vitro experiment of epithelial cells. The proliferated AT2 cells were apoptotic with a higher signal of regulation of DNA fragments or necrosis relative to the control cells. Furthermore, newborn AT2 cells showed high sensitivity to the tumor necrosis factor response. (Fig. 5D). GSEA was performed on the DEGs of epithelial cells. Nine-monthold coal-exposed AT2 epithelial cells exhibited declined outside-related transcription levels after mechanical stimulation. Moreover, the mitochondrial transport, protein coupling, and intracellular ATP increased (Fig. 5E), suggesting that energy metabolism disorder is a critical link in the toxic process of coal dust that leads to cell death.

AT2 epithelial cells are a few essential cells, accounting for only $5 \%$ of the total lung cells, but they can proliferate and differentiate into AT1 epithelial cells, which repair alveolar structures. After the coal dust treatment, the alveolar type II cells proliferated significantly, indicating that their repair function was activated. However, our transcriptomes disclosed that proliferated AT2 showed functional heterogeneity, with reduced antibacterial activity, functional loss, and abnormal proliferation. Thus, the space-occupying lesions of coal dust in the lung interstitium may have caused abnormal processes or initiated their reprogramming. Nevertheless, the timeseries analysis suggested that the newly generated AT2 epithelial cells were still highly active, which may be critical in inducing lung consolidation.

\section{Alveolar macrophages show rheostatic phenotypes and $\mathrm{M} 2$ polarization in mice with coal pneumoconiosis}

Mononuclear macrophages in the lung play a critical role in maintaining tissue homeostasis and controlling the 


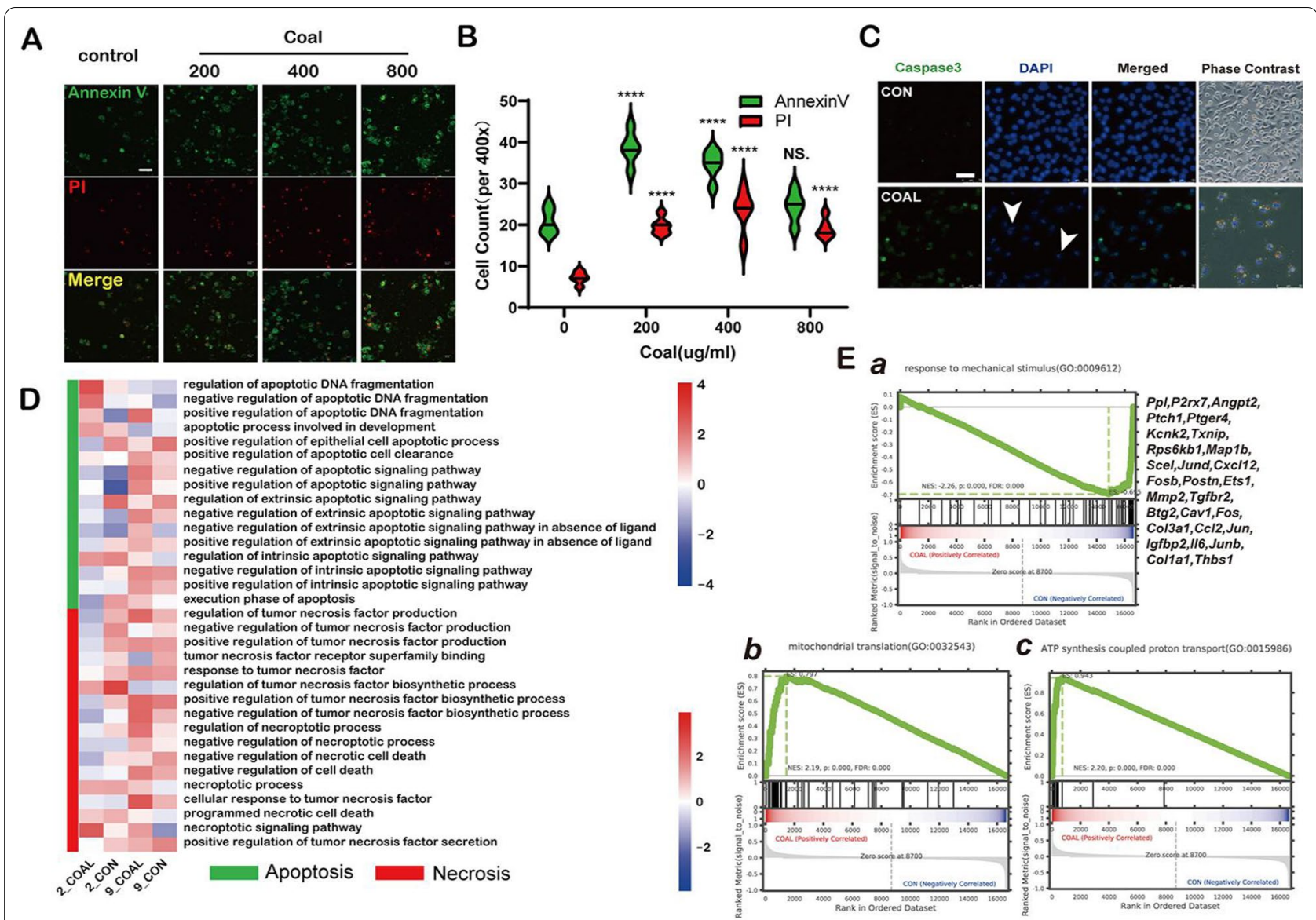

Fig. 5 Coal dust exposure-induced pulmonary apoptosis and necrosis. A The MLE-12 cells, a commonly used murine cell line expressing some type II alveolar epithelial cell markers, were either treated or not with coal dust $(0 \mu \mathrm{g} / \mathrm{mL}, 200 \mu \mathrm{g} / \mathrm{mL}, 400 \mu \mathrm{g} / \mathrm{mL}$, and $800 \mu \mathrm{g} / \mathrm{mL})$ for $24 \mathrm{~h}$. Microscopic fluorescent images were used to detect cell apoptosis or necrosis after annexin V/PI staining. Cells with the dual stain of annexin V/PI appear bright green in early apoptosis and red in late apoptosis, while the living and untreated (control) cells appear in a rare green color under a fluorescent microscope. B The counts of annexin V/PI positive cells (under a $400 \times$ magnification) at each dose are shown in the graph. C Representative image of cleaved caspase 3 (CCP3) after immunofluorescent staining revealed apoptotic green cells after coal dust exposure. The untreated cells showed a dark field with negative CCP3 staining. DAPI was used as a counterstain for the nucleus. Apoptosis induced by coal dust in MLE-12 cells presented nuclear degeneration with condensed and damaged chromatin indicated by the DAPI stain. Coal dust was colocalized with cells under bright fields. The data values are expressed as Mean \pm SEM. The data were derived from triplicate assays and two independent experiments. ${ }^{*} p<0.05$ or ${ }^{* *} p<0.05$ compared with the untreated cells. D Heatmap of the apoptosis and necrosis expression of type-Il alveolar epithelial cells in Gene Ontology (GO) terms. (E) GSEA plots of the top two differentially expressed regulons between coal dust ( $n=963$ cells) and PBS lungs ( $n=1900$ cells). The gene set enrichment analysis (GSEA) revealed that coal dust-exposed lungs highly enriched the ATP synthesis coupled proton of the collagen fibril organization and response to mechanical stimulus modules. The $p$-value was calculated using a permutation test (one-sided) based on phenotype, showing the statistical significance of the enrichment score

(See figure on next page.)

Fig. 6 After long-term coal dust exposure, the heterogeneity, phenotypic diversity, and functional alternation of pulmonary macrophages. A tSNE plot view of 3582 macrophages, color-coded by the associated cluster (top) or the assigned cell type (bottom). B The fraction of macrophage subclusters from each of the four mice, box plots of the number of cells (with the plot center corresponding to the median). C The relative increase or decrease of the macrophages of clusters in the coal-exposed lungs was observed, compared with the control (positive represents more, negative represents less); D Single-Cell RNA-SEQ identified seven macrophage populations in mouse lungs. The tSNE diagram shows an aggregation of 3582 cells from 4 mice. E The volcano plot of the SDEGs of pulmonary macrophages from the coal-exposed mice and the control. A total of 129 upregulated genes are presented in red, while 266 downregulated genes are presented in blue (bar chart). F Kyoto Encyclopedia of Genes and Genomes (KEGG) pathway classification map. The genes were divided into six branches according to the biological pathways. G The top 20 significantly pathways are enriched by KEGG based on significantly differentially expressed genes (SDEGs). HThe Gene Ontology (GO) enrichment analysis shows the association between the genes and phenotypes of coal dust-exposed mice 
A

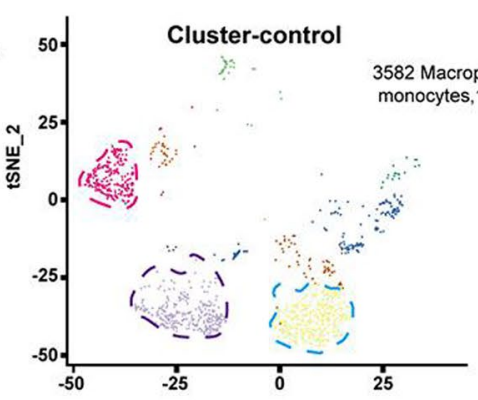

C

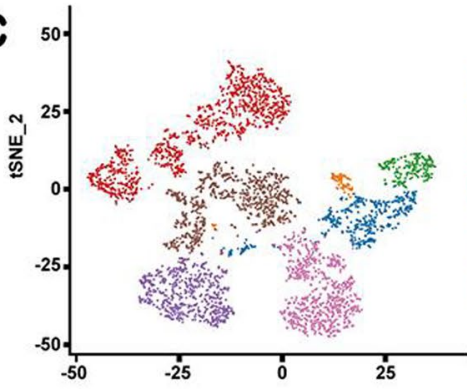

E

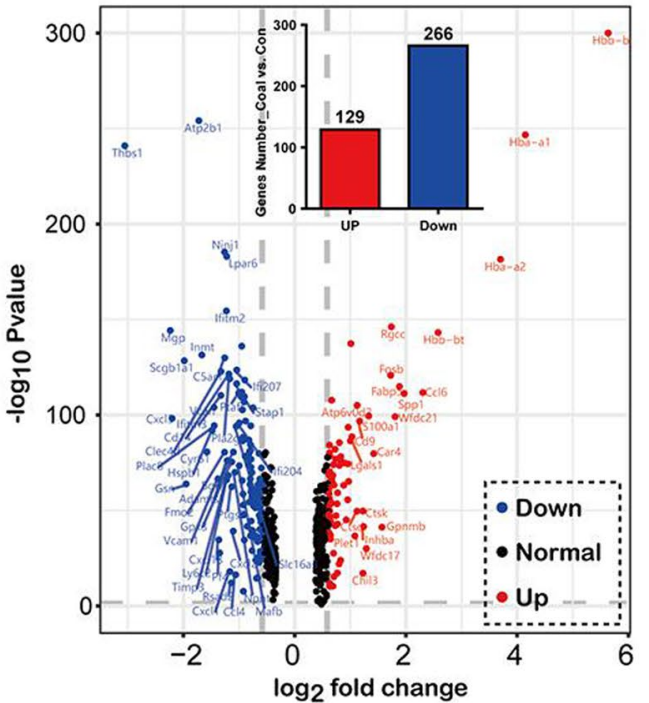

DC (DC.8-4-11B+)
Cluster-coal

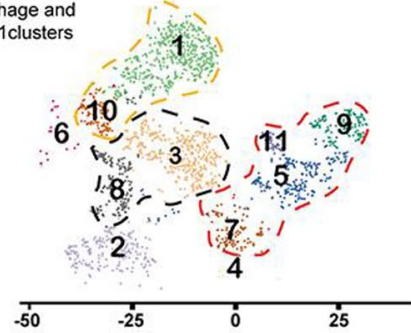

B
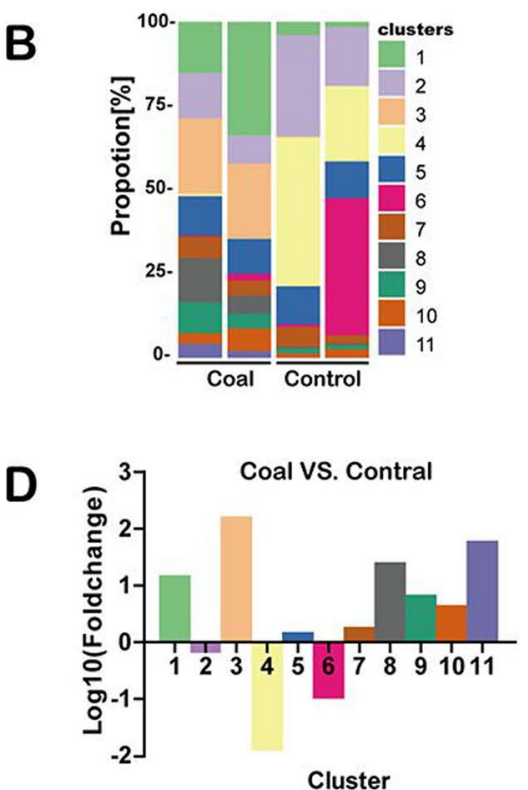

Cluster
DC (DC.103-11B+24+)

$\mathrm{DC}(\mathrm{DC} .8+)$

Macrophages (MF)

Macrophages (MF.103CLOSER)

Macrophages (MFIO5.II-480INT)

Monocytes (MO.6C+II-)

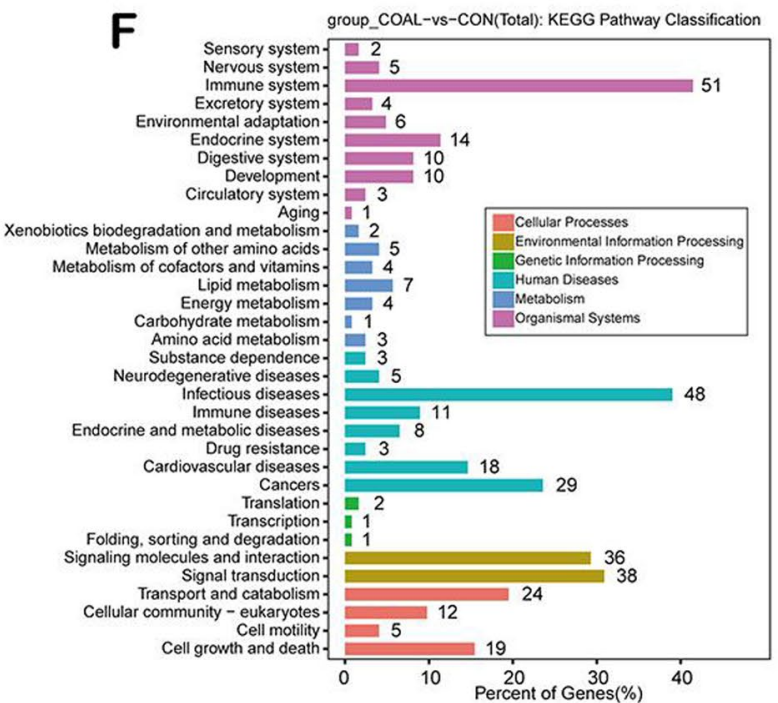

H

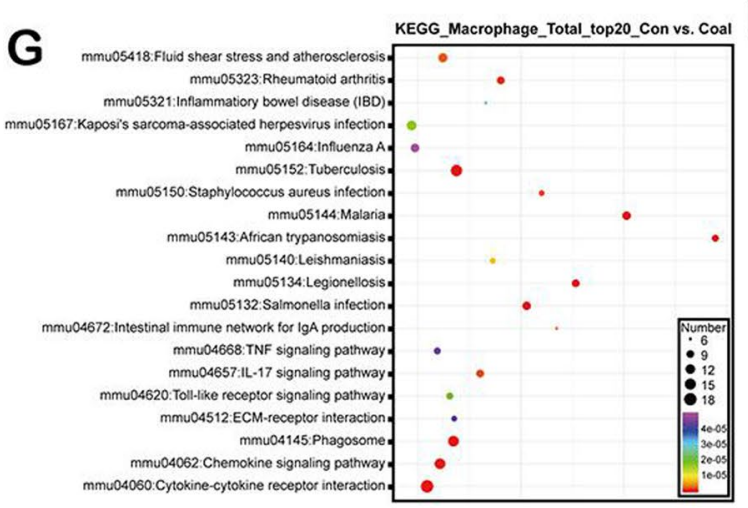
60:0002526GO:0042742- 3 GO:0030324GO:0002250 GO:0009611GO:0009611 GO:0006952-

o, GO:0070374-

GO:0007155- 17

23

GO:0071222 12

GO:0002548- 11

GO:0035313-

60:0043065-7 6

60

G0:0030855- 6

GO:0000082

GO:0030574- 4

GO:0070374

GO:0006954

regulation of 1 -kappaB kinase/NF-kappaB signaling defense response to bacterium acute inflammatory response

lung development

adaptive immune response

defense response

positive regulation of ERK1 and ERK2 cascade

cell adhesion

neutrophil chemotaxis

cellular response to lipopolysaccharide

inflammatory response

monocyte chemotaxis

8 wound healing, spreading of epidermal cells positive regulation of apoptotic process

regulation of inflammatory response

G1/S transition of mitotic cell

Girs transition or milobic cell cycie

positive regulation of ERK inflammatory response

padj $2.78 \mathrm{E}-19 \sim 0.0469325$

Fig. 6 (See legend on previous page.) 
inflammatory response to dust inhaled from the environment and lung disease pathogenesis [46]. To define the transcriptional landscape of lung-resident macrophages, we partitioned 3,582 transcriptionally distinct mononuclear macrophages into 11 clusters, which were shown in the coal dust and control groups (Fig. 6A). The cell-type composition of macrophages differed substantially in mice with coal pneumoconiosis (Fig. 6B, D). In all clusters, essential marker genes were highly expressed, while markers of other cell types were minimally expressed, except for OGN genes (Additional file 2: Fig. S2). A dissection of the heterogeneous origins of mouse alveolar macrophages revealed that these were macrophages, monocytes, and dendritic cells (Fig. 6A, bottom). The mixed profile of alveolar macrophages (AMs) represents phenotypic plasticity in response to inhaled coal dust. Here, two types of macrophages were detected in the normal mouse lung. It has been reported that AMs highly express the MARCO, FABP4, and MCEMP1 genes. The other type, interstitial macrophages, is derived from circulating monocytes. These cells are functionally different from tissue-resident macrophages, recruiting and expressing profibrotic genes during lung fibrosis [47]. Next, the AM type in lung tissues, including antiinflammatory $\mathrm{AM}\left(\mathrm{C} 2\right.$ and $8, \mathrm{APOE}^{+}, \mathrm{CD}_{163}{ }^{+}$, and $\left.\mathrm{C} \mathrm{qb}^{+}\right)$, pro-inflammatory $\mathrm{AM}\left(\mathrm{C} 10, \mathrm{IL} 1 \beta^{+}\right.$, and C6 $\mathrm{IL}^{+}$), and actively cycling AM expressing anti-inflammatory markers were found. Moreover, as expected, we confirmed the decline of macrophage type 1 (M1)/M2 in mice with 9-month (advanced-stage) coal pneumoconiosis, indicating the activation of adaptive anti-inflammatory repair in response to long-term coal dust exposure (Fig. 6C, Additional file 2: Fig. S2). Consistently, the lungs of mice with coal pneumoconiosis were strongly enriched in Macs (anti- and pro-inflammatory macrophages in clusters 1,3 , and 8). In addition, both normal lungs and those infected with coal pneumoconiosis contained clusters of $\mathrm{C} \mathrm{qb}^{+}$macrophages $(\mathrm{C} 2)$.

To investigate the functional role of coal dust-induced changes at the transcription levels in macrophages, we identified 395 significantly differentially expressed genes (SDEGs), which included 266 downregulated and 129 upregulated genes in the coal group relative to the control group (Fig. 6E, Additional file 8: Table S4). We marked the top 20 upregulated and downregulated genes between the coal and control groups according to the $\log _{2} \mathrm{FC}$ in Additional file 8: Table S2. Significantly downregulated genes included ATP2b1, Thbs1, Ninj1, Lpar6, Lfitm, Scgb1a1, Mgp, CD14, and Cxcl3. To further study the complex behavior of CMP macrophages, a pathway enrichment analysis of DEGs was applied to understand better the biological function of a gene and its interaction with others. The differential genes in the macrophages were involved in 37 subclasses of metabolic pathways in six broad categories. Next, the data analysis by the metabolic pathway database KEGG was performed (Fig. 6F). A high number of genes were found among these signaling pathways, including the immune system (51 genes), infectious diseases (48 genes), signal transduction (38 genes), cancers (29 genes), and cardiovascular diseases (18 genes). Metabolic pathways are involved in signal transduction during environmental changes.

Meanwhile, there were four pathways associated with immune and disease resistance, including signaling molecules and interaction (36 genes), transport and catabolism (24 genes), cell growth and death (19 genes), and cellular community-eukaryotes (12 genes). These pathways are involved in all aspects of mammalian immunization. In short, the experimental KEGG annotation pathway enrichment analyses for macrophages based on lineage-specific genes identified by scRNA-Seq strongly highlighted immune-induced regulatory pathways in the pathogenesis of coal pneumoconiosis in mice. This study's genes and pathways associated with the immune system, signal transduction, and disease processes were similar to those previously reported for particle-induced pneumoconiosis [48-50]. Thus, studies on immunerelated genes and pathways identification here can advance our understanding of the molecular immune mechanisms in coal dust-activated CMP.

KEGG pathway enriched and analyzed all important signal transduction pathways and biochemical metabolic pathways regulated by DEGs [51]. 196 KEGG pathways annotated SDEGs of coal group/control group, and the first 20 enrichment pathways are shown in Fig. 6G (Additional file 8: Table S5 in details). We demonstrated six significantly enriched KEGG pathways, including the phagosome, tuberculosis, cytokine-cytokine receptor interaction, chemokine signaling pathway, IL-17 signaling pathway, and Salmonella infection pathways. The most significant enrichment signal is the cytokine-cytokine receptor interaction pathway, including 18 SDEGs such as interferon-gamma receptor 1 (Ifngr1), Ccl17, Ccl24, Ccl4, Ccl6, Ccl9, Ccr5, Cxcl1, Cxcl10, Cxcl13, Cxcl2, $\mathrm{Cxcl} 3$, Cxcr4, and more. In addition, we confirmed that autophagosome markers LAMP2, LC3B, and Beclin1, which are related to phagocytic pathways, were mostly co-located or adjacent to M2 macrophages that phagocytic coal dust particles (Additional file 3: Fig. S3). The single-cell analysis-based dissection uncovered macrophage phenotype changes linked to functional state transitions in the 9-month coal-exposed mouse lungs.

The transcriptome of macrophages reveals changes responsible for immune defense and tissue repair. To reveal some of the pathways that might contribute to coal pneumoconiosis formation in macrophages, GO 
term analysis was performed on the DEGs of the CMP transcriptome to compare the results to the control group. The top 22 most significantly upregulated and downregulated transcripts are listed in Fig. 6H. Pathway analysis revealed that the most highly upregulated biological processes involved wound healing, the spread of epidermal cells (GO0035313), the positive regulation of the apoptotic process (GO0043065), epithelial cell differentiation (GO0030855), and collagen catabolic processes (GO003057). The repressed biological processes included processing in the regulation of 1-kappaB kinase/NF-kappaB signaling (GO0043122), defense response to bacteria (GO0002526), cell adhesion (GO0030593), and defense response (GO0009611).

The epithelial crosstalk with macrophage in mice with coal pneumoconiosis is predicted using ligand-receptor interaction analysis

As we have successfully outlined the fate of epithelial cells and macrophages and delineated the molecular characteristics of different cell populations, we then infer a common ligand-receptor database mediated intercellular communication in advanced coal dust lung disease. CellPhoneDB was developed to predict the key signaling events between the spatially co-located cell groups by Efremova et al. [42]. The CellPhoneDB analysis performed on different epithelial cell types, including endothelial cells, fibroblasts, macrophages, neutrophils, and lymphocytes, showed that macrophages were the dominant communication hubs that secrete and reverse signals via 262 and 316 ligand-receptor pairs (Fig. 7A, E). After comparing ligand-receptor pairs with cell-specific genes, we classified possible ligand-receptor pairs in different cell populations (Additional file 8: Table S6-1, 6-2). Regarding ligand-receptor pairs (epithelium and macrophage specification) in the control and CMP groups, we found a stronger interaction relationship between the epithelial cells and macrophages in CMP (Fig. 7B, C).

To further predict the toxic damage mechanism in CMP lungs, we studied the signaling correspondence between epithelia and macrophages. After coal dust treatment, these cells co-located in space and actively signaled to each other. There were significant positive and negative signal states in macrophages, indicating that the cell state transition under coal dust exposure was highly regulated. Specifically, the TNF pathway showed rich signaling interactions among all four states, with CD74 receptors expressed primarily by macrophages. In addition, the dominantly ligand CD44 was contributing to dermal TGF signaling, which promotes granuloma formation by stimulating the in situ proliferation of mononuclear cells through autocrine and paracrine signaling [52]. Furthermore, macrophages secreted SPP1 to multiple cell surface receptors, consistent with the known role of physiological and pathological processes, including wound healing, inflammation, tumorigenesis, and ischemia. In addition, epithelial cells were the major ligand source for another important Notch signaling pathway that primarily expresses autocrine Notch1 and Notch2 in CMP.

The Cell Phone DB analysis also predicted that this dominant macrophage notch signaling was supplemented by a minor epithelial-derived Nothch2 ligand paracrine signaling, promoting macrophage M2 polarization, dependent on the interaction with CD47 and mediated by intracellular signaling through SHP-1 $[53,54]$. Our analysis showed solid intercellular communication between macrophages and epithelial cells, including the innate immune system signaling members SIRPA and $\mathrm{CD} 47$, and Notch signaling ligands Jag1, gag2, Tgfb2, and Fam3c (Fig. 7D). These results further emphasize the indispensable roles of these well-defined pathways during coal dust lung specification. In addition, we observed robust ligand-receptor pairs within the epithelial cells and macrophage population, including SSP1, TGF-b, and $\mathrm{CD} 74$, indicating a robust autocrine relationship at this stage (Fig. 7C). However, some of these ligand-receptor pairs differed between epithelial cells and macrophages, suggesting heterogeneity in coal dust lung cells, as illustrated in our DEG analysis.

The infiltrated M2-type alveolar macrophages with CD206 ${ }^{+}$ and $\mathrm{MLPH}^{+}$subset after the long-term coal exposure were significantly decreased after Vitamin $D$ treatment

Next, we focused on markers of specialized biological features of macrophages. Using scRNA-Seq, the marker gene expression of macrophage clusters was shown in Additional file 4: Fig. S4 and Additional file 8: Table S7. The specialized M2 phenotypes expressing MLPH markers were found, further illustrating the specific M2 subset (Fig. 8B). Finally, alveolar macrophages in CMP lungs were polarized into the M2-type subset (Fig. 8A), and a fraction of this population was identified with double $\mathrm{CD}^{206}{ }^{+} \mathrm{MLPH}^{+}$expression in vivo and in vitro. In addition, the scRNA-Seq showed that 9-month coal-dust exposed lung tissues had increased M2 cells and relatively decreased M1 cells compared to control groups; however, this trend was reversed in the $\mathrm{VD}_{3}$-treated group (Fig. 8C).

Here, we focused on CD86, Mrc1, and MLPH for two reasons. First, CD68 and Mrc1 represent two different identities of the classical macrophage classification of M1 and M2 [55]. Second, scRNA-Seq data suggested that the MLPH expression was explicitly increased in alveolar macrophages at 9-month CMP, but only increased in M2 cells. Using selective marker genes (CD86 for M1 


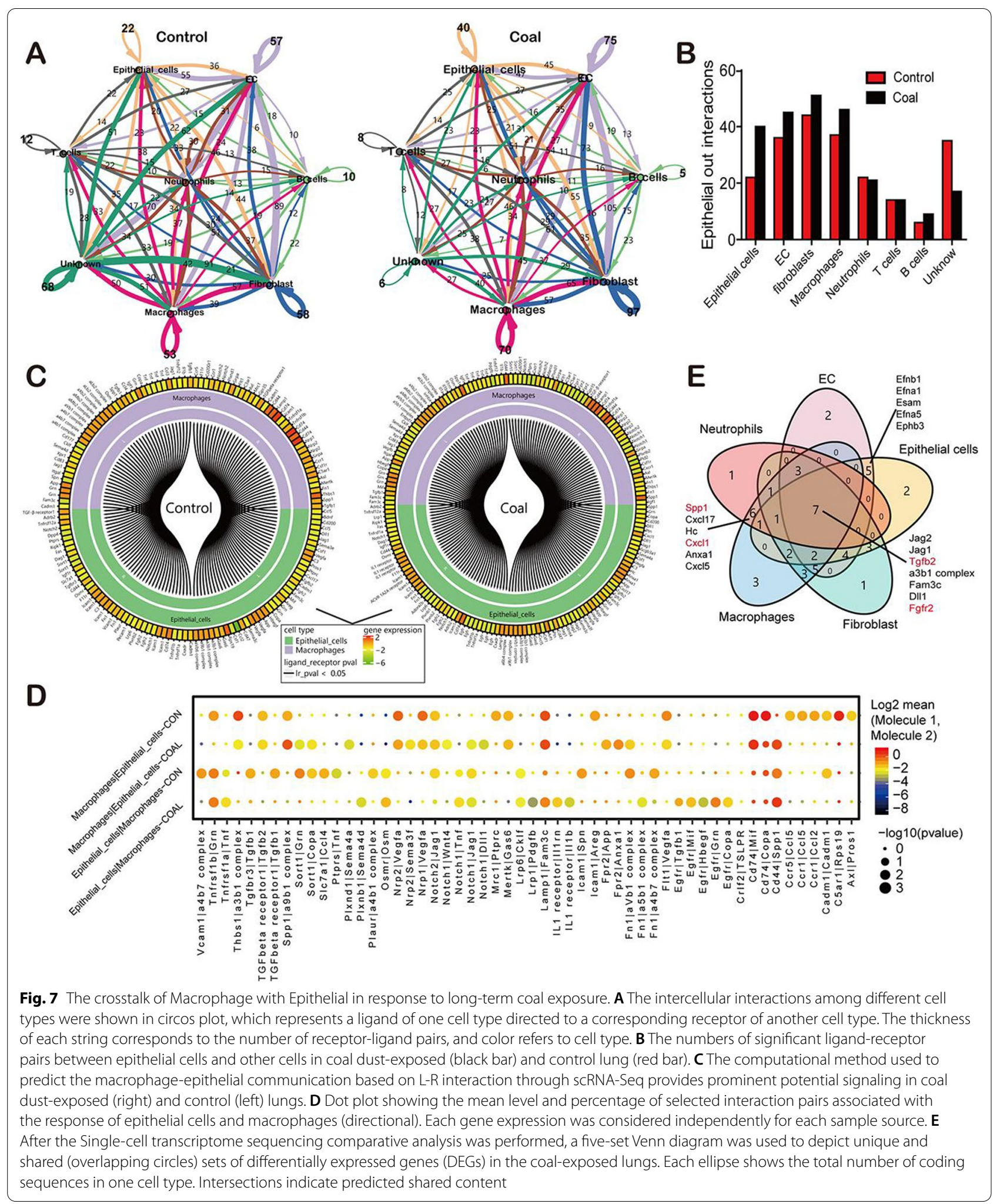




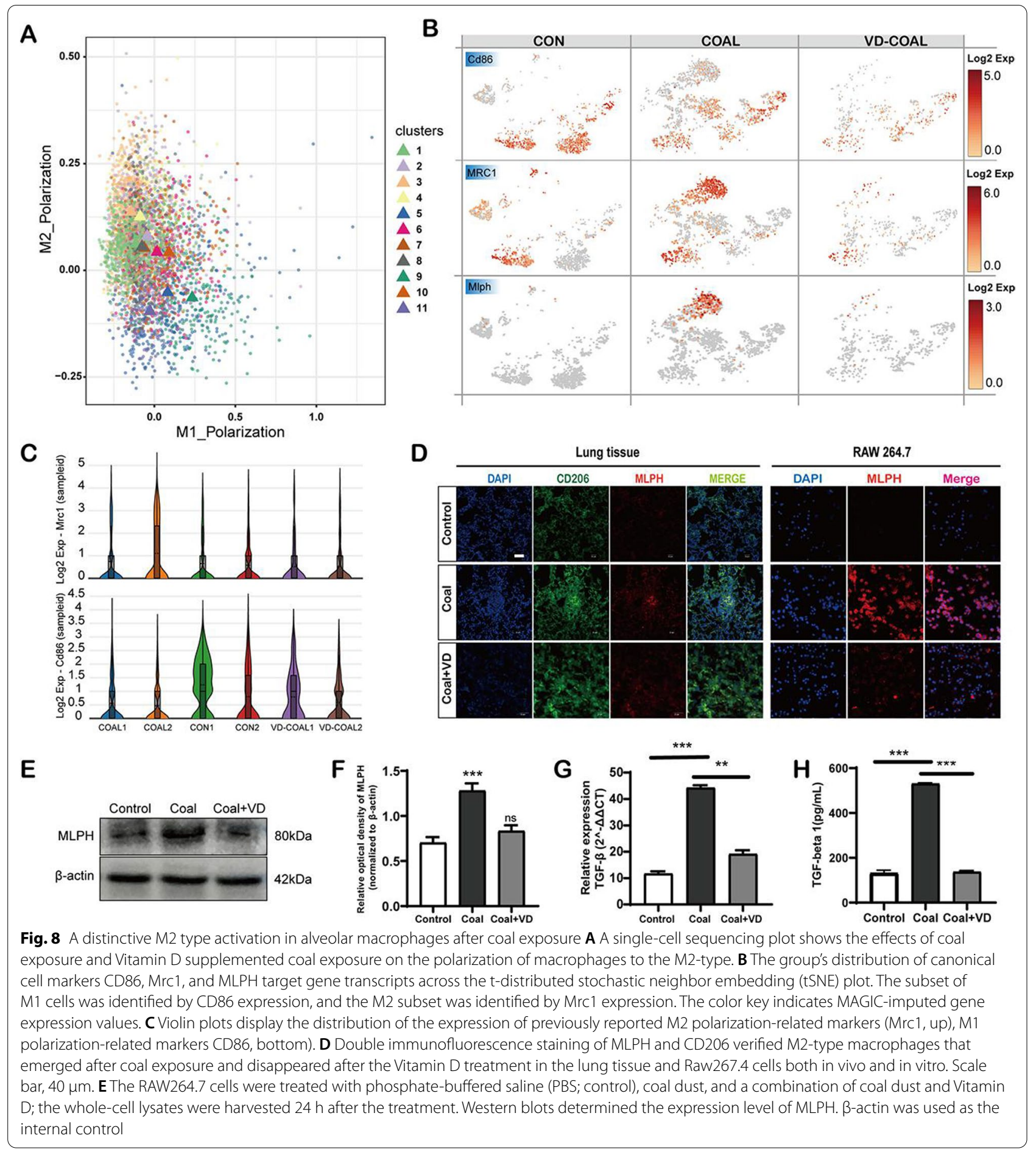

and Mrc1 for M2), we were able to confirm the predicted emergence of $\mathrm{MLPH}^{+}$macrophages and upregulated in M2 cells only in CWP lungs (Fig. 8B). Vitamin D exerts its beneficial effects on many macrophage components, modulating phagocytic activity and cytokine production [56]. To validate that $\mathrm{VD}_{3}$ accurately regulated gene expression in specific macrophage populations, we performed in situ immunofluorescent staining (Fig. 8D) and western blot (Fig. 8E, F) of the samples from the same tissues used for single-cell RNA-Seq analysis. The result showed that the MLPH expressed in CMP lungs increased significantly more than in the control lungs but 
markedly decreased after the Vitamin D treatment. Furthermore, the qPCR and ELISA analysis revealed that coal-exposed macrophages had increased TGF- $\beta$ expression and secretion but decreased after the $\mathrm{VD}_{3}$ treatment (Fig. 8G, H). TGF- $\beta$ plays a critical role in lung inflammation and is a recognized indicator of fibrosis. Our results suggest that $\mathrm{VD}_{3}$ may be a potential alternative treatment for alleviating pulmonary fibrosis caused by coal dust. These results also support the feasibility of the supplement of the $\mathrm{VD}_{3}$ in patients with coal pneumoconiosis.

\section{Discussions}

Coal dust-induced alveolar structural damage and repair are major challenges in lung disease. Although it has been reported that AT2 epithelial cells have significant regeneration capacity, this regeneration process leads to abnormal or stunted repair [12, 57]. Herein, we provide new insights into the single-cell analysis. A new population of HAAS emerged in the long-term coal-exposed mouse lungs, which lost antibacterial pathways, including CHI3L1, APOC1, CXCL15, IL33, and LYZ1, and played an important role in pulmonary immunity. IL-33 is a significant driver of cellular differentiation and lung maturation, produced mainly in AT2 cells [58], which binds uniquely to IL1rl1 in adjacent basophils of lung tissue. The proportion of AT2 epithelial cells was small in normal lungs, but there was an abnormal increase after coal dust exposure. Furthermore, newborn AT2 cells produce more disease-associated factors, such as IL33. IL-33 specifically primes basophils and mast cells and affects AM maturation toward M2-type polarization [59, 60]. IL33 is also a marker of repair promotion and is expressed in AT2 during lung development and maturation [61]. It is also a significant factor that regulates hypoxia-induced lung injury and can mediate hypoxia-inducible factors [62]. Fate-mapping studies indicate a clear lineage relationship between AT2 and HAAS. Therefore, we amended the hierarchy of alveolar epithelial cells involving these convertible cell states. Specific gene expression signatures demonstrate that HAAS gradually loses AT2 characteristics and represents a unique convertible population. The HAAS of the human lung has been previously identified and is especially apparent in the fibrotic regions of IPF lungs. Regarding human HAAS cells, HAAS-like cells in the present murine lungs were characterized by enrichment genes related to cellular senescence, Notch signaling, and WNT-regulated genes, all of which are linked to lung fibrosis [25, 63]. In contrast, we found a high expression of TRP63, KRT5, and COL1A1 in the epithelial cells of mice with coal pneumoconiosis; these were previously found only in human HAAS cells.
We found that AT2 cells undergo broad stimulation and deformation after coal dust entering the alveoli, causing them ready for DNA damage, a feature of most lung diseases with pulmonary fibrosis [12]. Previous studies have revealed that respirable particles lead to DNA damage once they move or remain in the pulmonary interstitium [64-66]. Our in vivo and in vitro study suggests that mouse AT2 cells undergo extensive stimulation and deformation during exposure to coal dust particles. Therefore, this specific AT2 cell state induced by the coal dust implies DNA damage in lung diseases.

The lungs efficiently match air and blood within the vast area of the alveolar surface, ensuring oxygen intake and delivery. Coal dust induced-hypoxia is a crucial driver of Notch signaling activation in epithelial stem/ progenitor cells. It has previously been reported that hypoxia can induce Notch activity in vitro [67]. Our single-cell sequencing analysis showed that coal pneumoconiosis activates Notch activity. Using CellphoneDB, we found that anoxic/notch-activated signals in the epithelium and macrophages communicate with a group of genes controlling motility and invasion. Nevertheless, taken together, these data paint a pneumoconiosis epithelium and macrophage picture suggestive of more preventative, coactive, and reparative roles on their part, crucial in maintaining lung function and homeostasis. Future studies are required to characterize the potential of alveolar progenitors to drive a regenerative alveolar process and decode the immune microenvironment that favors AT2 proliferation.

A combination of single-cell transcriptome sequencing and immunofluorescence studies confirmed that disease-relevant $\mathrm{MLPH}^{+}$macrophages are connected by a branched lineage that proceeds from coal dustinduced M2 polarization. We found that the homeostatic alveolar macrophages in mice rarely contained the $\mathrm{MLPH}^{+}$subset but expressed highly CD86. However, coal exposed-alveolar macrophages had a large proportion of the $\mathrm{CD}_{206}{ }^{+} \mathrm{MLPH}^{+}$double staining subset, which may ultimately lead to pulmonary fibrosis. Interestingly, MLPH is involved in intracellular substance transport and becomes an upstream complex of TGF- $\beta$ signaling. In addition, we deconstructed the VD3 supplement by scRNA-Seq as a comprehensive model and analyzed the effects of VD3 on macrophages to the subset level. Our scRNA-Seq data suggest that the coalinduced macrophage M2 polarization is suppressed by $\mathrm{VD}_{3}$ supplementation, which defines $\mathrm{VD}_{3}$ as a key to homeostatic M1/M2 of murine macrophages in a manner that involves other activities but does not exclude antifibrosis. This important function of $\mathrm{VD}_{3}$ in macrophage therapy may benefit from their hormone activity alone or 
their ability to coordinate both paracellular and transcellular transport activity across the other cells.

Taken together, we uncovered a highly active AT2 state (HAAS), an abnormal increase that spans between AT2 and AT1. Notable, we further identified coal pneumoconiosis macrophage niches and clarified multiple functional enrichment pathways for alveolar macrophages, through which activated alveolar macrophages facilitate two-way communication with epithelial cells. Our results indicated M2-type macrophage polarization in long-term coal-exposed mouse lungs and disclosed an M2-macrophage subset with $\mathrm{MLPH}^{+} \mathrm{CD}^{206^{+}}{ }^{\text {expression that significantly decreased }}$ after the administration of the Vitamin $\mathrm{D}_{3}$ treatment. These findings provide potential alternative targets for CWP in the future. This study was based on a mouse model of coal pneumoconiosis. Next, we will validate the molecular mechanism discovered by single cell sequencing in human pneumoconiosis samples.

\section{Conclusions}

This study successfully established a reliable coal pneumoconiosis mouse model to study the cytotoxicity of respiratory coal dust particles to lung epithelial cells and macrophages in coal mines. After 9 months of coal dust stimulation, pulmonary inflammation and pulmonary fibrosis were significantly induced. Singlecell transcriptome sequencing technology allows the characterization of whole lung cells and molecules and provides a more comprehensive picture of coal-dustinduced lung cell changes and possible signal communication than traditional mechanism studies. Our study is the first to show that chronic coal dust stimulation can lead to substantial heterogeneity of epithelial cells and macrophages, and induce cellular inflammatory and death pathways, thereby promoting the progression of pulmonary fibrosis. Among them, HAAS, the highly active state of epithelial cells with no antibacterial ability, suggested the repair imbalance of lung epithelial cells. In addition, a subset of M2-type macrophages generated by the polarization of M2 macrophages induced by coal dust could be inhibited by VD, which may be related to the alleviation of the pulmonary fibrosis process by VD.

\footnotetext{
Abbreviations

AMs: Alveolar macrophages; AP: Alkaline phosphatase; AT1: Alveolar type 1 cells; AT2: Alveolar type 2 cells; BMDMs: Bone marrow-derived macrophages; CCP3: Cleaved caspase 3; CMP: Coal pneumoconiosis in mice; CWP: Coal worker's pneumoconiosis; DEGs: Differentially expressed genes; GEMs: Gel beads in emulsions; GO: Gene Ontology; GSEA: Gene set enrichment analysis; GSVA: Gene set variation analysis; HAAS: Highly active AT2 state; HE: Hematoxylin and eosin; IHC: Immunohistochemistry; PBST: Phosphate buffered
}

saline with Tween; BSA: Bovine serum albumin; ATP: Adenosine triphosphate; AAT2: Actived alveolar epithelial cell type 2; CDPs: Coal dust particles; FN: Fibronectin; RFs: Repair related factor; BEC: Blood endothelial cells; ExCM: Extracellular matrix; IM: Interstitial macrophages; KEGG: Kyoto Encyclopedia of Genes and Genomes; KRT5: Keratin 5; M1: Macrophage type 1; M2: Macrophage type 2; MSHA: Mine Safety and Health Administration; NS: Normal saline; PBS: Phosphate-buffered saline; PCA: Principal component analysis; PI: Propidium iodide; PMF: Progressive massive fibrosis; PVDF: Polyvinylidene difluoride; SCRNA-SEQ: Single-cell RNA sequencing; sCRNAseq: Single-cell transcriptomics; SDEGs: Significantly DEGs; SPC: Surfactant protein C; tSNE: T-distributed stochastic neighbor embedding; qPCR: Quantitative real-time PCR; UMI: Unique molecular identifier.

\section{Supplementary Information}

The online version contains supplementary material available at https://doi. org/10.1186/s12989-022-00449-y.

Additional file 1. Figure S1. Macrophage annotation and identification. (A) Visualized transcriptome cluster by tSNE. (B) Expression levels of the indicated genes projected onto tSNE in Clasters10,11,17. (C) Overview of violin plot for expression of critical lineage-associated genes by macrophages (number of cells in each cluster displayed in B).

Additional file 2. Figure S2. Distribution of two subsets of functional macrophages types across tSNE plot. Macrophages expressed pro-inflammatory $\left(\mathrm{CD}^{207^{+}}, \mathrm{IL} 1 \beta^{+}, \mathrm{TNF}^{+}, \mathrm{IL} 6^{+}\right)$and anti-inflammatory $\left(\mathrm{IL} 10^{+}, \mathrm{APOE}^{+}\right.$, $\left.\mathrm{CD} 63^{+}, \mathrm{Clab}^{+}\right)$genes.

Additional file 3. Figure S3. IHC analysis of CD206, Beclin1, LC3B, LAMP2 in coal dust-exposed lung. Data values represent (Mean \pm SEM) obtained from two independent experiments in triplicate assays. The graph showed the quantification of intensity when comparing the sections from the coal group with the control ones $\left({ }^{*} p<0.05,{ }^{*} p<0.01,{ }^{*} p<0.001\right)$ : scale bar, $50 \mu \mathrm{m}$.

Additional file 4. Figure S4. The heat map showed the top 10 markers of macrophage.

Additional file 5. Figure S5. Pulmonary score and Lung function test of mice exposed to coal dust for nine months. (A) Pathology score performed by Roderick J. Pathology classification standard of lung injury at three, six, nine-months. (B) Pulmonary fibrosis score performed by Ashcroft Criteria for grading lung fibrosis at three, six, nine -months. (C) Body weight recorded throughout the nine months. (D) The main indexes of lung function are Penh: enhanced pause; Mv: minute volume; Av: accumulated volume; EF50: expiratory flow 50\%; PIF: peak inspiratory flow; PEF: peak expiratory flow; F: frequency; Rpef: ratio of time to peak expiratory flow $\left({ }^{*} p<0.05,{ }^{* *} p<0.01,{ }^{* *} p<0.001\right)$.

Additional file 6. Figure S6. Toxic effects of coal dust on MLE-12 cells. Causing $50 \%$ inhibition of cell survival (CC50) of coal dust was measured in MLE-12 cells treated with different dose (A) and different time point at $200 \mu \mathrm{g} / \mathrm{ml}(\mathrm{B})\left({ }^{*} P<0.05,{ }^{* *} P<0.01,{ }^{* * *} P<0.001\right.$, Treatment vs Control).

Additional file 7. Advanced analytical methods.

Additional file 8. Raw datasets.

\section{Acknowledgements}

Not applicable.

\section{Authors' contributions}

Manuscript writing: MM, BL, WYW, HBC, QXS; Formal analysis: YJZ, HMC, DYG, Conceptualization, Methodology: JHW, XRT, YL; Editing: HHT, DH. All authors read and approved the final manuscript. We thank OE Biotech Co., Ltd (Shanghai, China) for providing single-cell RNA-seq, Xiaohua Yao for assistance with bioinformatics analysis.

\section{Funding}

This study was supported by the Anhui University Science Research Project (No. KJ2019ZD13, No. KJ2019A0131) and The University Synergy Innovation Program of Anhui Province (GXXT-2021-077). 


\section{Availability of data and materials}

All data related to this study are publicly available upon reasonable request to the corresponding author.

\section{Declarations}

Ethical approval and consent to participate

The Biomedical Research Ethics Committee of Anhui University of Science and Technology approved our study (No. 2020006).

\section{Consent for publication}

Not applicable.

\section{Competing interests}

The authors declare that they have no competing interests.

\section{Author details}

${ }^{1}$ Key Laboratory of Industrial Dust Control and Occupational Health of the Ministry of Education, Anhui University of Science and Technology, Huainan, China. ${ }^{2}$ Key Laboratory of Industrial Dust Deep Reduction and Occupational Health and Safety of Anhui Higher Education Institutes, Anhui University of Science and Technology, Huainan, China. ${ }^{3}$ School of Medicine, Department of Medical Frontier Experimental Center, Anhui University of Science and Technology, 168 Taifeng Road, Huainan, China. ${ }^{4}$ Anhui Province Engineering Laboratory of Occupational Health and Safety, Anhui University of Science and Technology, Huainan, China. ${ }^{5}$ Cancer Institute, Fudan University Shanghai Cancer Center, Fudan University, Shanghai, China.

Received: 30 September 2021 Accepted: 13 January 2022 Published online: 20 January 2022

\section{References}

1. Prasad SK, Singh S, Bose A, Prasad B, Banerjee O, Bhattacharjee A, et al. Association between duration of coal dust exposure and respiratory impairment in coal miners of West Bengal, India. Int J Occup Saf Ergon: JOSE. 2021;27(3):794-804. https://doi.org/10.1080/10803548.2020.17424 63.

2. Lancet. Improving occupational health in China. Lancet. 2019;394 10197:443; doi: https://doi.org/10.1016/S0140-6736(19)31799-4

3. Zosky GR, Hoy RF, Silverstone EJ, Brims FJ, Miles S, Johnson AR, et al. Coal workers' pneumoconiosis: an Australian perspective. Med J Aust. 2016:204 11:414-8

4. Perret JL, Plush B, Lachapelle P, Hinks TS, Walter C, Clarke P, et al. Coal mine dust lung disease in the modern era. Respirology. 2017;22 4:662-70; doi: https://doi.org/10.1111/resp.13034.

5. Leon-Mejia G, Silva LF, Civeira MS, Oliveira ML, Machado M, Villela IV, et al. Cytotoxicity and genotoxicity induced by coal and coal fly ash particles samples in V79 cells. Environ Sci Pollut Res Int. 2016;23 23:24019-31; doi: https://doi.org/10.1007/s11356-016-7623-z.

6. Leon-Mejia G, Machado MN, Okuro RT, Silva LFO, Telles C, Dias J, et al. Intratracheal instillation of coal and coal fly ash particles in mice induces DNA damage and translocation of metals to extrapulmonary tissues. Sci Total Environ. 2018:625:589-99. https://doi.org/10.1016/j.scitotenv.2017.12.283.

7. Attfield MD, Seixas NS. Prevalence of pneumoconiosis and its relationship to dust exposure in a cohort of U.S. bituminous coal miners and ex-miners. Am J Ind Med. 1995;27 1:137-51.

8. Centers for Disease C, Prevention. Pneumoconiosis and advanced occupational lung disease among surface coal miners--16 states, 2010-2011. MMWR Morbidity and mortality weekly report. 2012;61 23:431-4.

9. MSHA: Respirable Coal Mine Dust Rule Enters Final Phase of Implementation. 2016.

10. Vlahovich KP, Sood A. A 2019 update on occupational lung diseases: a narrative review. Pulm Ther. 2021;7(1):75-87. https://doi.org/10.1007/ s41030-020-00143-4.

11. Kania N, Setiawan B, Widjadjanto E, Nurdiana N, Widodo MA, Kusuma HMSC. Subchronic inhalation of coal dust particulate matter 10 induces bronchoalveolar hyperplasia and decreases MUC5AC expression in male Wistar rats. Exp Toxicol Pathol. 2014;66(8):383-9. https://doi.org/10.1016/j. etp.2014.06.001
12. Kobayashi Y, Tata A, Konkimalla A, Katsura H, Lee RF, Ou J, et al. Persistence of a regeneration-associated, transitional alveolar epithelial cell state in pulmonary fibrosis. Nat Cell Biol. 2020;22(8):934-46. https://doi.org/10. 1038/s41556-020-0542-8

13. Fu JH, Xue XD, Pan L, Xu W. Expression of HoxB5 mRNA and their effect on lung development in premature rats with hyperoxia-induced chronic lung disease. Zhonghua Er Ke Za Zhi. 2008;46(7):540-3.

14. Davies LC, Jenkins SJ, Allen JE, Taylor PR. Tissue-resident macrophages. Nat Immunol. 2013;14(10):986-95. https://doi.org/10.1038/ni.2705.

15. Rom WN. Role of oxidants in interstitial lung diseases: pneumoconioses, constrictive bronchiolitis, and chronic tropical pulmonary eosinophilia. Mediators Inflamm. 2011;2011: 407657. https://doi.org/10.1155/2011/407657.

16. Tang J, Diao P, Shu X, Li L, Xiong L. Quercetin and quercitrin attenuates the inflammatory response and oxidative stress in LPS-induced RAW264.7 cells: in vitro assessment and a theoretical model. Biomed Res Int. 2019:2019:7039802; doi: https://doi.org/10.1155/2019/7039802.

17. Travaglini KJ, Nabhan AN, Penland L, Sinha R, Gillich A, Sit RV, et al. A molecular cell atlas of the human lung from single-cell RNA sequencing. Nature. 2020;587(7835):619-25. https://doi.org/10.1038/ s41586-020-2922-4.

18. Treutlein B, Brownfield DG, Wu AR, Neff NF, Mantalas GL, Espinoza FH, et al. Reconstructing lineage hierarchies of the distal lung epithelium using single-cell RNA-seq. Nature. 2014;509(7500):371-5. https://doi.org/ 10.1038/nature13173.

19. Rosen C, Shezen E, Aronovich A, Klionsky YZ, Yaakov Y, Assayag M, et al. Preconditioning allows engraftment of mouse and human embryonic lung cells, enabling lung repair in mice. Nat Med. 2015:21(8):869-79. https://doi.org/10.1038/nm.3889.

20. Kamer I, Daniel-Meshulam I, Zadok O, Bab-Dinitz E, Perry G, Feniger-Barish $\mathrm{R}$, et al. Stromal-MDM2 promotes lung cancer cell invasion through tumor-host feedback signaling. Mol Cancer Res. 2020;18(6):926-37. https://doi.org/10.1158/1541-7786.MCR-19-0395.

21. Angelidis I, Simon LM, Fernandez IE, Strunz M, Mayr CH, Greiffo FR, et al. An atlas of the aging lung mapped by single cell transcriptomics and deep tissue proteomics. Nature communications. 2019;10 1:963; doi: https://doi.org/10.1038/s41467-019-08831-9.

22. Liu X, Qin X, Qin H, Jia C, Yuan Y, Sun T, et al. Characterization of the heterogeneity of endothelial cells in bleomycin-induced lung fibrosis using single-cell RNA sequencing. Angiogenesis. 2021. https://doi.org/10.1007/ s10456-021-09795-5.

23. Chakarov S, Lim HY, Tan L, Lim SY, See P, Lum J, et al. Two distinct interstitial macrophage populations coexist across tissues in specific subtissular niches. Science. 2019;363 6432; doi: https://doi.org/10.1126/science. aau0964.

24. Nakahara Y, Hashimoto N, Sakamoto K, Enomoto A, Adams TS, Yokoi T, et al. Fibroblasts positive for meflin have anti-fibrotic property in pulmonary fibrosis. Eur Respir J. 2021. https://doi.org/10.1183/13993003.03397-2020.

25. Xu Y, Mizuno T, Sridharan A, Du Y, Guo M, Tang J, et al. Single-cell RNA sequencing identifies diverse roles of epithelial cells in idiopathic pulmonary fibrosis. JCl insight. 2016;1 20:e90558; doi: https://doi.org/10.1172/ jci.insight.90558.

26. Ongaratto R, Marostica P, Pinto LA. Association between hypovitaminosis $D$ and frequency of pulmonary exacerbations in children and adolescents with cystic fibrosis. Pediatr Pulm. 2016;51:S40-1.

27. Sexauer WP, Hadeh A, Ohman-Strickland PA, Zanni RL, Varlotta L, Holsclaw $D$, et al. Vitamin D deficiency is associated with pulmonary dysfunction in cystic fibrosis. J Cyst Fibros. 2015;14(4):497-506. https://doi.org/10.1016/j. jcf.2014.12.006.

28. Peng $M$, Chen SH, Jiang $X J$, Zhang WG, Wang $Y X$, Wu SL, et al. Dissociation between low vitamin $D$ level and hypertension in coal mine workers: evidence from the Kailuan study. Internal Med. 2016;55(10):1255-60. https:// doi.org/10.2169/internalmedicine.55.5898.

29. Sarikaya S, Ozdolap S, Mungan AG, Gumustas S, Koc U, Guven B, et al. Effect of underground working on vitamin D levels and bone mineral densities in coal miners: a controlled study. J Int Med Res. 2006;34(4):3626. https://doi.org/10.1177/147323000603400404

30. Hanel A, Neme A, Malinen M, Hamalainen E, Malmberg HR, Etheve S, et al. Common and personal target genes of the micronutrient vitamin $D$ in primary immune cells from human peripheral blood. Sci Rep-Uk. 2020;10 1; doi: https://doi.org/10.1038/s41598-020-78288-0. 
31. Pirdel $\mathrm{L}$, Pirdel M. A differential immune modulating role of vitamin $\mathrm{D}$ in urinary tract infection. Immunol Invest. 2020:1-15; doi: https://doi.org/10. 1080/08820139.2020.1845723.

32. Tao SS, Zhang $H$, Xue L, Jiang XY, Wang HY, Li BY, et al. Vitamin D protects against particles-caused lung injury through induction of autophagy in an Nrf2-dependent manner. Environ Toxicol. 2019;34(5):594-609. https:// doi.org/10.1002/tox.22726.

33. Li B, Mu M, Sun Q, Cao H, Liu Q, Liu J, et al. A suitable silicosis mouse model was constructed by repeated inhalation of silica dust via nose. Toxicol Lett. 2021;353:1-12. https://doi.org/10.1016/j.toxlet.2021.09.014.

34. Szarka R, Wang N, Gordon L, Nation P, Smith R. A murine model of pulmonary damage induced by lipopolysaccharide via intranasal instillation. J Immunol Methods. 1997;202(1):49-57. https://doi.org/10.1016/s00221759(96)00236-0.

35. Ashcroft T, Simpson J, Timbrell V. Simple method of estimating severity of pulmonary fibrosis on a numerical scale. J Clin Pathol. 1988;41(4):467-70. https://doi.org/10.1136/jcp.41.4.467.

36. Chen S, Zhu G, Yang Y, Wang F, Xiao YT, Zhang N, et al. Single-cell analysis reveals transcriptomic remodellings in distinct cell types that contribute to human prostate cancer progression. Nat Cell Biol. 2021;23(1):87-98. https://doi.org/10.1038/s41556-020-00613-6.

37. Mabbott NA, Baillie JK, Brown H, Freeman TC, Hume DA. An expression atlas of human primary cells: inference of gene function from coexpression networks. BMC Genomics. 2013;14:632. https://doi.org/10.1186/ 1471-2164-14-632.

38. Zhang P, Yang M, Zhang Y, Xiao S, Lai X, Tan A, et al. Dissecting the singlecell transcriptome network underlying gastric premalignant lesions and early gastric cancer. Cell Rep. 2019;27(6):1934-47. https://doi.org/10. 1016/j.celrep.2019.04.052.

39. Hanzelmann S, Castelo R, Guinney J. GSVA: gene set variation analysis for microarray and RNA-seq data. BMC Bioinformatics. 2013;14:7. https://doi. org/10.1186/1471-2105-14-7.

40. Reimand J, Isserlin R, Voisin V, Kucera M, Tannus-Lopes C, Rostamianfar A et al. Pathway enrichment analysis and visualization of omics data using g:Profiler, GSEA. Cytoscape EnrichmentMap Nat Protoc. 2019:14(2):482517. https://doi.org/10.1038/s41596-018-0103-9.

41. Butler A, Hoffman P, Smibert P, Papalexi E, Satija R. Integrating single-cell transcriptomic data across different conditions, technologies, and species. Nat Biotechnol. 2018;36(5):411-20. https://doi.org/10.1038/nbt.4096.

42. Trapnell C, Cacchiarelli D, Grimsby J, Pokharel P, Li S, Morse M, et al. The dynamics and regulators of cell fate decisions are revealed by pseudotemporal ordering of single cells. Nat Biotechnol. 2014;32(4):381-6. https://doi.org/10.1038/nbt.2859.

43. Patel AP, Tirosh I, Trombetta JJ, Shalek AK, Gillespie SM, Wakimoto H, et al. Single-cell RNA-seq highlights intratumoral heterogeneity in primary glioblastoma. Science. 2014;344(6190):1396-401. https://doi.org/10.1126/ science.1254257.

44. Vento-Tormo R, Efremova M, Botting RA, Turco MY, Vento-Termo M, Meyer KB, et al. Single-cell reconstruction of the early maternal-fetal interface in humans. Nature. 2018;563 7731:347. doi: https://doi.org/10.1038/s41586-018-0698-6.

45. Efremova M, Vento-Tormo M, Teichmann SA, Vento-Tormo R. Cell PhoneDB: inferring cell-cell communication from combined expression of multi-subunit ligand-receptor complexes. Nat Protoc. 2020;15(4):1484506. https://doi.org/10.1038/s41596-020-0292-x.

46. Hume PS, Gibbings SL, Jakubzick CV, Tuder RM, Curran-Everett D, Henson PM, et al. Localization of macrophages in the human lung via designbased stereology. Am J Respir Crit Care Med. 2020;201(10):1209-17. https://doi.org/10.1164/rccm.201911-21050C

47. Kim N, Kim HK, Lee K, Hong Y, Cho JH, Choi JW, et al. Single-cell RNA sequencing demonstrates the molecular and cellular reprogramming of metastatic lung adenocarcinoma. Nature communications. 2020;11 1:2285; doi: https://doi.org/10.1038/s41467-020-16164-1.

48. Leung CC, Yu IT, Chen W. Silicosis. Lancet. 2012;379 9830:2008-18; doi: https://doi.org/10.1016/S0140-6736(12)60235-9.

49. Maeda M, Nishimura Y, Kumagai N, Hayashi H, Hatayama T, Katoh M, et al. Dysregulation of the immune system caused by silica and asbestos. J Immunotoxicol. 2010;7(4):268-78. https://doi.org/10.3109/1547691X. 2010.512579

50. Vlahovich KP, Sood A. A 2019 update on occupational lung diseases: a narrative review. Pulmonary Ther. 2021;7(1):75-87. https://doi.org/10. 1007/s41030-020-00143-4.
51. Kanehisa M, Sato Y, Furumichi M, Morishima K, Tanabe M. New approach for understanding genome variations in KEGG. Nucleic Acids Res. 2019;47(D1):D590-5. https://doi.org/10.1093/nar/gky962.

52. Schmits R, Filmus J, Gerwin N, Senaldi G, Kiefer F, Kundig T, et al. CD44 regulates hematopoietic progenitor distribution, granuloma formation, and tumorigenicity. Blood. 1997;90(6):2217-33.

53. Lin X, Wang S, Sun M, Zhang C, Wei C, Yang C, et al. miR-195-5p/NOTCH2mediated EMT modulates IL-4 secretion in colorectal cancer to affect M2-like TAM polarization. J Hematol Oncol. 2019;12 1:20; doi: https://doi. org/10.1186/s13045-019-0708-7.

54. Lin Y, Zhao JL, Zheng QJ, Jiang X, Tian J, Liang SQ, et al. Notch signaling modulates macrophage polarization and phagocytosis through direct suppression of signal regulatory protein alpha expression. Front Immunol. 2018;9:1744. https://doi.org/10.3389/fimmu.2018.01744.

55. Soldano S, Trombetta AC, Contini P, Tomatis V, Ruaro B, Brizzolara R, et al. Increase in circulating cells coexpressing M1 and M2 macrophage surface markers in patients with systemic sclerosis. Ann Rheum Dis. 2018;77(12):1842-5. https://doi.org/10.1136/annrheumdis-2018-213648.

56. Bergandi L, Apprato G, Silvagno F. Vitamin D and beta-glucans synergically stimulate human macrophage activity. Int J Mol Sci. 2021;22 9; doi: https://doi.org/10.3390/ijms22094869.

57. Choi J, Park J-E, Tsagkogeorga G, Yanagita M, Koo B-K, Han N, et al. Inflammatory signals induce AT2 cell-derived damage-associated transient progenitors that mediate alveolar regeneration. Cell Stem Cell. 2020;27 3; doi: https://doi.org/10.1016/j.stem.2020.06.020.

58. de Kleer IM, Kool M, de Bruijn MJW, Willart M, van Moorleghem J, Schuijs $\mathrm{MJ}$, et al. Perinatal activation of the interleukin-33 pathway promotes type 2 immunity in the developing lung. Immunity. 2016;45(6):1285-98. https://doi.org/10.1016/j.immuni.2016.10.031.

59. Starkey MR, McKenzie AN, Belz GT, Hansbro PM. Pulmonary group 2 innate lymphoid cells: surprises and challenges. Mucosal Immunol. 2019;12(2):299-311. https://doi.org/10.1038/s41385-018-0130-4.

60. Fanny M, Nascimento M, Baron L, Schricke C, Maillet I, Akbal M, et al. The IL-33 receptor ST2 regulates pulmonary inflammation and fibrosis to bleomycin. Front Immunol. 2018;9:1476. https://doi.org/10.3389/fimmu.2018.01476.

61. Marega M, Chen C, Bellusci S. Cross-talk between inflammation and fibroblast growth factor 10 during organogenesis and pathogenesis: lessons learnt from the lung and other organs. Front Cell Dev Biol. 2021;9: 656883. https://doi.org/10.3389/fcell.2021.656883.

62. Liu J, Wang W, Wang L, Chen S, Tian B, Huang K, et al. IL-33 initiates vascular remodelling in hypoxic pulmonary hypertension by up-regulating HIF-1 $a$ and VEGF expression in vascular endothelial cells. EBioMedicine. 2018;33:196-210. https://doi.org/10.1016/j.ebiom.2018.06.003.

63. Chanda D, Otoupalova E, Smith SR, Volckaert T, De Langhe SP, Thannickal VJ. Developmental pathways in the pathogenesis of lung fibrosis. Mol Aspects Med. 2019;65:56-69. https://doi.org/10.1016/j.mam.2018.08.004.

64. Wu R, Högberg J, Adner M, Ramos-Ramírez P, Stenius U, Zheng H. Crystalline silica particles cause rapid NLRP3-dependent mitochondrial depolarization and DNA damage in airway epithelial cells. Part Fibre Toxicol. 2020;17 1:39; doi: https://doi.org/10.1186/s12989-020-00370-2.

65. Khaliullin TO, Kisin ER, Guppi S, Yanamala N, Zhernovkov V, Shvedova AA. Differential responses of murine alveolar macrophages to elongate mineral particles of asbestiform and non-asbestiform varieties: cytotoxicity, cytokine secretion and transcriptional changes. Toxicol Appl Pharmacol. 2020;409: 115302. https://doi.org/10.1016/j.taap.2020.115302.

66. Valavanidis A, Vlachogianni T, Fiotakis K, Loridas S. Pulmonary oxidative stress, inflammation and cancer: respirable particulate matter, fibrous dusts and ozone as major causes of lung carcinogenesis through reactive oxygen species mechanisms. Int J Environ Res Public Health. 2013;10(9):3886-907. https://doi.org/10.3390/ijerph10093886.

67. Chen J, Imanaka N, Chen J, Griffin JD. Hypoxia potentiates Notch signaling in breast cancer leading to decreased E-cadherin expression and increased cell migration and invasion. Br J Cancer. 2010;102(2):351-60. https://doi.org/10.1038/sj.bjc.6605486.

\section{Publisher's Note}

Springer Nature remains neutral with regard to jurisdictional claims in published maps and institutional affiliations. 